“C 2018 IEEE. Personal use of this material is permitted. Permission from IEEE must be obtained for all other uses, in any current or future media, including reprinting/republishing this material for advertising or promotional purposes, creating new collective works, for resale or redistribution to servers or lists, or reuse of any copyrighted component of this work in other works." 


\title{
Modeling a Composite Molecular Communication Channel
}

\author{
Muneer M. Al-Zu'bi, Student Member, IEEE, and Ananda S. Mohan, Senior Member, IEEE
}

\begin{abstract}
The paper deals with modelling of propagation medium for molecular communication, which consist of composite biological environments with multiple regions each with distinct diffusion properties for the understanding of the transport of information molecules. For this, we propose a generalized analytical approach for modelling a composite, diffusive, molecular communication channel for arbitrary placement of the transmitting and receiving nano-machines. Using this approach, we derive a generalised closed-form expression for the channel impulse response of a three dimensionally (3D) diffusive medium having multiple regions with an aim to act as a benchmark solution to validate simulations as well as to optimize the design of molecular communication systems. The pulse peak amplitude, pulse peak time and pulse width are derived to evaluate the system performance. In addition, a particle-based simulator for modelling a diffusive medium with multiple regions under three-dimensional diffusion is proposed and validated with the analytical results. It is shown that the channel impulse response and other communication metrics are significantly affected by the diffusion coefficients, regions thickness, properties of region interfaces as well as the positions of transmitter and receiver nano-machines with respect to the interfaces.
\end{abstract}

Index Terms-Bio-nano machine, channel impulse response, composite medium, diffusion, molecular communication, multiple regions.

\section{INTRODUCTION}

$\mathrm{M}$ OLECULAR communication (MC) is a new communication paradigm in which the information is exchanged through chemical molecules (e.g., ions, proteins, DNA etc.,) [1]-[2]. The biocompatibility and low energy requirement makes it highly suitable for applications involving engineered biological systems where the use of electromagnetic (EM) waves is neither possible nor desirable [3]-[4].

In the literature, various forms of molecular communication have been described viz., molecular communication via diffusion (MCvD) [5], bacterium-based communication [6], and microtubules [7] etc. Among these, the MCvD offers a simple method of propagating information molecules from a transmitter bio-nano machine (TN) to a receiver bio-nano machine (RN) that are located in a fluidic biological medium, without requiring additional infrastructure or external energy [2]. The information molecules propagate in the medium (i.e.,

This work is supported in part by UTS FEIT Bluesky Research Grant.

M. Al-Zu'bi and A. S. Mohan are with the Centre for Health Technologies, Faculty of Engineering and IT, University of Technology Sydney, NSW, 2007, Australia (e-mail: \{Muneer.Al-zubi, Ananda.Sanagavarapu\}@uts.edu.au). propagation channel) following the Brownian motion and the number of received molecules can be estimated by the channel impulse response (CIR) [8]-[9]. The CIR also helps to estimate other channel characteristics that may be required for the design of the bio nano-machines and/or suitable equalization and detection schemes [10]-[11].

The propagation channel usually is the aqueous cellular medium inside a human body consisting of biological tissues, blood vessels, etc., that helps with the transport of information molecules. The most common and simplifying assumption on the modelling of the molecular propagation channel that has widely been employed in the MCvD literature, is to consider it as a single homogeneous region with constant viscosity and medium temperature which thus, lead to constant diffusion coefficients, [3]-[5], [10]-[11], [12]. However, in nature, stratified or composite cellular environments with multiple regions each with different and distinct properties exist in which molecules may propagate from a source to a destination. Some of the examples of molecular propagation in stratified composite biological media include the transport through the cell membrane consisting of almost entirely of a lipid bilayer [13], the diffusion of oxygen from the alveolus into the red blood cells, diffusion of carbon dioxide in the opposite direction throughout the layers of the respiratory membrane [14], transport of drugs across the blood-brain barrier [15], and the medium involved with Drug-Eluting Stents (DES) [16].

In general, for modelling such composite $\mathrm{MCvD}$ environments, the CIR is required for obtaining both the diffusive channel characteristics and the variation of molecular concentration levels with time [17]. Almost all cells in a biological in vivo environment are surrounded by other cells as well as extracellular matrix (ECM) and it is possible that the molecules can diffuse in any arbitrary direction [18]. Thus, it is important to model the molecular channel considering diffusion in all three directions (3-D). A training-based estimation technique to estimate the CIR for MC based on the observed number of molecules at the receiver using Poisson distribution was presented in [9]. However, this work examined the performance of different estimators for a homogeneous medium of single diffusion coefficient without providing any insight 
about modelling a multiple-region medium. Recently, in the literature, the channel impulse response (CIR), delay spread etc., have been derived for a MCvD medium with multiple regions with different diffusion properties by replacing it with an equivalent homogeneous medium having a single region with an effective diffusion properties [19]-[20]. The effective (average) diffusion coefficient is derived in [19]-[20] by averaging the diffusion coefficients of all the multiple regions. However, the assumptions under which the average diffusion coefficient was derived in [19]-[20] are valid only for onedirectional diffusion and under steady-state when the time derivative of molecular concentration is equal to zero [21]. Then the authors substitute this average diffusion coefficient in a simple concentration expression for homogeneous medium of single region, i.e., [19, Eq. 11]. Moreover, simple averaging approach of the diffusion coefficients of a multiple region biological medium is not accurate, as it does not properly model the effect of interfaces between various regions on the molecular diffusion. In particular, it fails to include the specific nature of these interfaces, which may be either fully or partially permeable.

Thus, there is a need to derive expressions for CIR for accurately modelling the stratified, composite molecular propagation media. In the mathematical theory of diffusion, any diffusion problem can be solved for either transient or steadystate. However, the transient analysis provides more information on the channel characteristic with time. It is important to know how the channel impulse response or concentration profile changes within a short time and not just at very large time (i.e., at steady state). For example, in drug delivery system, it is important to analyze how the released drug molecules can affect the targeted site with time. Thus, we can stop delivery of drug and measure the toxic level with time. Moreover, in communication nanonetworks, the transient modeling is required to estimate the various characteristics of the channel such as inter-symbol inference (ISI), delay spread, and other metrics. It is important to realize that the derivation of closed form expressions even for a two-region MCvD channel can be significantly more complex than the simple averaging approach in [19]-[20]. The challenges are mainly posed by the finite, non-zero time derivatives of the concentration as well as the boundary conditions, which must be satisfied at the interfaces between different regions that may have different diffusion properties. In addition, obtaining exact analytical expressions for CIR for such scenarios are necessary, as they can act as reference solutions with which simulations and any other approximations can be compared. As far as simulations for modelling the multi-region molecular channel are concerned, the authors in [19]-[20] reported the use of existing 'N3Sim' simulator [22]. However, the 'N3Sim' simulator can only simulate a homogeneous medium only and cannot handle composite molecular communication channels with multiple regions. Hence, researchers resort to averaging approach to derive effective single region channel model and as a consequence compromise with poorer predictions which in turn can adversely affect the design a molecular communication system to operate in a multiple region channel and particularly, if the receiver ( $\mathrm{RN})$ uses peak amplitude detection technique [11]. Hence, there is also a need for the development of a composite, multiple-region channel simulator that can model molecular diffusion in any arbitrary direction.

In view of its significance, in this paper, we propose, a rigorous mathematical approach for obtaining generalised channel impulse response (CIR) for composite MCvD channel involving three-dimensional (3-D) diffusion. In this work, we model the molecular diffusion between the transmitter and receiver in multiple-region channel using molecular communication paradigm. We first derive closed-form analytical expressions for CIR of a composite molecular communication channel having multiple regions with differing diffusion properties and fully permeable interfaces considering arbitrary three directional (3-D) diffusion. The aim here is to present a benchmark, reference solution with which the simulations can be verified and validated as well as to evaluate various system performance metrics. We then propose a 3-D particle-based simulator for a composite MCvD medium and verify simulations with exact results for composite molecular channels having two, three, and four regions respectively. Further, the three important communication metrics, viz., pulse peak amplitude, pulse peak time, and pulse width are evaluated for different values of system parameters. To the best of our knowledge, neither the closed-form exact analytical model nor any particle-based simulator for $\mathrm{MCvD}$ in three-dimensionally (3-D) diffusive, multiple region medium are available yet, in the literature.

The rest of this paper is organized as follows. In Section II, we propose a generalised mathematical formulation for the channel impulse response (CIR), and then we derived exact closed form analytical expressions for CIR for a two-region and multiple region diffusive medium. The metrics for assessing the communication performance are discussed in section III. In section IV, a comprehensive simulation framework for multiple region, 3-D diffusive molecular channels is presented which is then verified with results computed using analytical expressions. Finally, we highlight the key contributions in section V.

\section{SYSTEM MODEL}

The system considered in this paper includes a transmitter bio nano-machine $(\mathrm{TN})$ communicating to a receiver bio nanomachine $(\mathrm{RN})$ to transfer information using chemical molecules via a composite, molecular diffusive propagation channel, which has multiple regions as shown in Fig.1. Each region is assumed to have different diffusion properties. The information molecules emitted by TN are assumed to diffuse independently in all three directions (3-D) within the propagation channel following Brownian motion to reach the RN. Here, we consider a cylindrical coordinate system to obtain mathematical solution for the proposed molecular propagation channel. We assume that the axis of the cylinder coincides with the z-axis and its radius goes to infinity. This channel consists of different 
regions $\Omega \mathrm{i}$, and each region except first and last regions is assumed to have a finite thickness $\delta \mathrm{i}$ with different viscosities and/or temperatures resulting in different diffusion coefficients $\mathrm{Di}$, and different molecular concentrations, $\mathrm{Pi}$, for $\mathrm{i}=1,2,3, \ldots, \mathrm{N}$. The thicknesses of first and last regions are assumed to be semiinfinite.

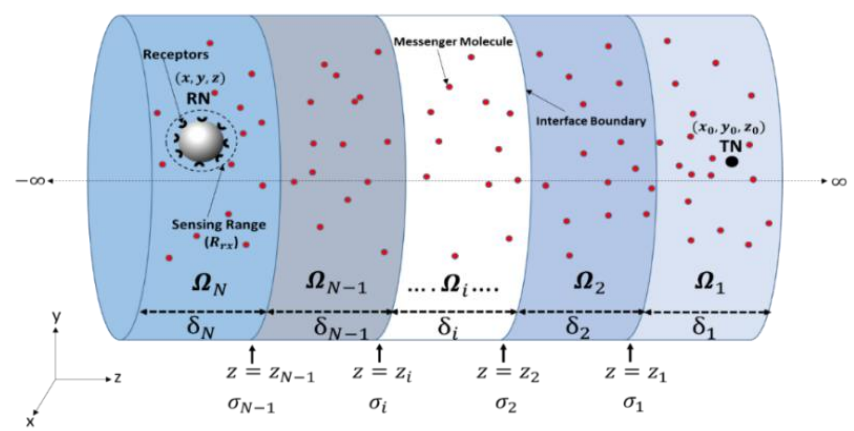

Fig. 1. MCvD in a composite propagation medium that has multiple regions.

There are $(N-1)$ interface boundaries between the regions that have permeability coefficients $\sigma_{j}$ at the locations $z_{j}$ for $\mathrm{j}=1,2,3, \ldots, N-1$. Furthermore, we assume that an instantaneous point-like TN is located in the region-1 $\left(\Omega_{1}\right)$ at the position $\left(x_{0}\right.$, $\left.y_{0}, z_{0}\right)$ and releases ' $Q$ ' information molecules into the channel at time $t=t_{0}$. The size of TN is assumed negligible compared with the relative distance ' $d$ ' between centres of TN and RN. Moreover, the RN is assumed to be a 3-D passive sphere with radius $R_{r x}$ and volume $V_{r x}$ and it is located at any general position $(x, y, z)$ in any of the regions. It is also assumed that the $\mathrm{RN}$ can count the number of received molecules at any time instant and does not restrict the movement of the information molecules. The information molecules are chemical compounds such as Insulin, which are assumed to diffuse independently carrying information from $\mathrm{TN}$ to $\mathrm{RN}$ following Brownian motion.

\section{A. Mathematical Formulation for Composite (Multiple-Region) Medium}

In this work, we assume each region to be isotropic meaning that the diffusion properties within each region to be same and invariant irrespective of the diffusion direction. The diffusion of molecules in a fluidic medium where they move independently from each other without collisions is described by the Fick's second law of diffusion [21] as

$$
\frac{\partial P_{i}\left(r, t ; r_{0}, t_{0}\right)}{\partial t}=D_{i} \nabla^{2} P_{i}\left(r, t ; r_{0}, t_{0}\right)
$$

where, $P_{i}\left(r, t ; r_{0}, t_{0}\right)$ is the molecular concentration for $i^{\text {th }}$ region in $\left(\mathrm{mol} / \mathrm{m}^{3}\right)$ at the location $r=(x, y, z)$ and at time $t$ due to molecules initially emitted at location $r_{0}=\left(x_{0}, y_{0}, z_{0}\right)$ and at time $t_{0}, \nabla^{2}$ is the Laplacian operator, and $D_{i}$ is the diffusion coefficient of $i^{\text {th }}$ region in $\left(\mathrm{m}^{2} / \mathrm{s}\right)$ given by [23],

$$
D_{i}=\frac{K_{B} T_{a}}{6 \pi \eta_{i} r_{m}}
$$

where, $K_{B}$ is the Boltzmann constant, $T_{a}$ is the medium absolute temperature in (Kelvin), $\eta_{i}$ is the dynamic viscosity constant of the $i^{\text {th }}$ fluid region, and $r_{m}$ is the hydraulic radius of the information molecules.

Employing a similar mathematical approach as in [24], we derive the channel impulse response (CIR) for the propagation channel with multiple regions. The expression for molecular concentration for $i^{\text {th }}$ region can be represented as a superposition of two functions in the Laplace-Transformed domain as

$$
\breve{P}_{i}=\breve{u}_{i}+\breve{v}_{i}
$$

where, the function $\breve{u}_{i}$ is the Laplace transform of the molecular concentration $u_{i}$ for a single, unbounded, 3-D isotropic diffusive medium received at a passive $\mathrm{RN}$ due to instantaneous TN that is given by [25]-[26],

$$
u_{i}=\frac{1}{\left(4 \pi D_{i}\left(t-t_{0}\right)\right)^{3 / 2}} \exp \left(-\frac{\left\|r-r_{0}\right\|^{2}}{4 D_{i}\left(t-t_{0}\right)}\right), \quad t \geq t_{0}
$$

where, the distance between $\mathrm{TN}$ and $\mathrm{RN}$ is $d=\left\|r-r_{0}\right\|=$ $\sqrt{R^{2}+\left(z-z_{0}\right)^{2}}$ and $R=\sqrt{\left(x-x_{0}\right)^{2}+\left(y-y_{0}\right)^{2}}$ is the radial distance.

The parameter $\breve{v}_{i}$ is the solution of the diffusion equation (5) that vanishes at $t=t_{0}$ and together with $\breve{u}_{i}$ enables (3) to satisfy the boundary conditions (6)-(9). Assuming that the diffusion to be symmetrical about the z-axis in a cylindrical coordinate system and is independent of the azimuth angle $\theta$, the Laplace transform of the diffusion equation [21, eq. (1.7)] for $i^{\text {th }}$ region can be expressed as

$$
\frac{\partial^{2} \breve{v}_{i}}{\partial R^{2}}+\frac{1}{R} \frac{\partial \breve{v}_{i}}{\partial R}+\frac{\partial^{2} \breve{v}_{i}}{\partial z^{2}}-\lambda_{i}^{2} \breve{v}_{i}=0
$$

where, the function $\breve{v}_{i}$ is the Laplace transform of the concentration $v_{i}, \lambda_{i}^{2}=s / D_{i}$, and "s" is a complex frequency parameter.

The boundary conditions at the interface between tworegions [21], [27] are extended for $\mathrm{N}$ regions medium in the Laplace domain:

$$
\begin{aligned}
& D_{j} \frac{\partial \breve{P}_{j}}{\partial z}=D_{j+1} \frac{\partial \breve{P}_{j+1}}{\partial z}, \quad z=z_{j} \\
& D_{j} \frac{\partial \breve{P}_{j}}{\partial z}=\sigma_{j}\left(\breve{P}_{j}-k_{j} \breve{P}_{j+1}\right), \quad z=z_{j}
\end{aligned}
$$

The boundary condition (6) imposes continuity of flux at the interface between the regions where the flux entering the interface equal to the flux flowing out. Moreover, it ensures that there is no accumulation of molecules at the interface between the regions assuming that the thickness of interface to be negligible. The boundary condition (7) allows for possible changes in molecular concentration at the interfaces in relation to the permeability of the interfaces via the permeability coefficient $\sigma_{j}$. The change in the concentration at each interface is equal to the difference between the concentrations on the both 
sides of that interface and the flux is also proportional to that. The permeability determines ability of the interface to allow the molecules to pass through it to the adjacent region. As special case, when $\sigma_{j} \rightarrow \infty$, the interface becomes fully permeable where all the molecules pass through it without any physical or chemical reactions.

We assume that the concentration for first and last regions vanishes at infinity and thus the corresponding boundary conditions in the Laplace domain turnout to be

$$
\begin{aligned}
& \lim _{z \rightarrow \infty} \breve{P}_{1}=0 \\
& \lim _{z \rightarrow-\infty} \breve{P}_{N}=0
\end{aligned}
$$

However, for fully permeable interfaces between regions (i.e., $\sigma_{j} \rightarrow \infty$ ), the molecules can move across the interfaces without any physical or chemical reactions and thus the condition (7) can be reduced to

$$
\breve{P}_{j}=k_{j} \breve{P}_{j+1}, \quad z=z_{j}
$$

The constants $k_{j}$ are the ratios of the uniform concentration in the region $\Omega_{\mathrm{j}}$ to that in region $\Omega_{\mathrm{j}+1}$ when final equilibrium is attained [21].

The transmitter $(\mathrm{TN})$ releases information molecules at time $t_{0}$ in the form of an impulsive signal and thus the initial condition in the Laplace domain can be expressed as

$$
\lim _{t \rightarrow t_{0}} \breve{P}_{i}= \begin{cases}\delta\left(r-r_{0}\right) e^{-s t_{0},} & i=1 \\ 0, & i=2,3, \ldots, N\end{cases}
$$

where, $\breve{P}_{i}$ is the Laplace transform of $P_{i}$ and $\delta($.$) is the Dirac$ delta function.

However, $\breve{u}_{i}$ vanishes for all regions except in the first region since a single point-like source (TN) is assumed to be present in that region. Now, the Laplace transform of concentration for each region can be expressed as

$$
\breve{P}_{i}=\left\{\begin{array}{cl}
\breve{u}_{i}+\breve{v}_{i}, & i=1 \\
\breve{v}_{i}, & i=2,3, \ldots, N
\end{array}\right.
$$

The term $\breve{v}_{i}$ for $i^{\text {th }}$ region can be expressed as follows [24]:

$$
\breve{v}_{i}=\int_{0}^{\infty}\left[A_{i}(\xi, s) e^{-g_{i} z}+B_{i}(\xi, s) e^{g_{i} z}\right] J_{0}(\xi R) \xi d \xi
$$

where, $A_{i}(\xi, s)$ and $B_{i}(\xi, s)$ are weighting functions, $J_{0}($.$) is the$ Bessel function of the first kind of order zero, and

$$
\begin{aligned}
& g_{i}=\left(\xi^{2}+\lambda_{i}^{2}\right)^{1 / 2} \\
& \lambda_{i}=\left(s / D_{i}\right)^{1 / 2}
\end{aligned}
$$

By taking the Laplace transform of (4) using [28, eq. (29.3.82)], $\breve{u}_{i}$ can be obtained as

$$
\breve{u}_{1}=\frac{e^{-\lambda_{1} \sqrt{R^{2}+\left(z-z_{0}\right)^{2}}} e^{-s t_{0}}}{4 \pi D_{1} \sqrt{R^{2}+\left(z-z_{0}\right)^{2}}}
$$

Following the procedure given in Appendix A, $\breve{u}_{1}$ can be expressed as

$$
\breve{u}_{1}=\frac{e^{-s t_{0}}}{4 \pi D_{1}} \int_{0}^{\infty} \frac{1}{g_{1}} e^{-g_{1}\left|z-z_{0}\right|} J_{0}(\mathrm{R} \xi) \xi d \xi
$$

In general, for an N-region channel, the functions $A_{i}(\xi, s)$ and $B_{i}(\xi, s)$ can be obtained by solving the system of $2(N-1)$ equations (6)-(7). Therefore, increasing the number of regions will increase the complexity of derivation of the CIR, unlike in the steady state case [19]-[20].

The channel impulse response is defined as the expected number of received molecules by $\mathrm{RN}$ at time $t$ after "Q" molecules were released instantaneously by TN at time $t=t_{0}$ [9], [29]. It is common to assume that the receiver is sufficiently far from the transmitter i.e., $d \gg R_{r x}$. Under this assumption, the molecule concentration throughout the volume of the passive $\mathrm{RN}$ can be considered to be uniform and equal to that expected at its centre [29]. Based on this assumption, the channel impulse response can be concluded directly from [9, Eq. 1] or [29, Eq. 20] and can be expressed as

$$
\bar{N}_{i}=Q V_{r x} P_{i}, \quad i=1,2, \ldots, N
$$

where, $P_{i}$ is the concentrations in $i^{\text {th }}$ region given by the inverse Laplace transform of (12), and $V_{r x}=(4 / 3) \pi R_{r x}^{3}$ is the volume of the spherical RN. For a homogeneous diffusive medium of infinite extent, given that TN emits "Q" molecules instantaneously at time $t=t_{0}$, the CIR can be expressed by replacing $P_{i}$ in (18) by the concentration of an infinite homogeneous medium given by (4).

The values of $k_{j}$ can be obtained after attaining the equilibrium when the concentrations in the regions do not change with time and the time derivative of concentrations vanishes i.e., $\partial P_{1} / \partial t=\partial P_{2} / \partial t=\cdots=$ $\partial P_{N} / \partial t=0$. Knowing that $D_{j} \nabla^{2} P_{j}=D_{j+1} \nabla^{2} P_{j+1}$ from (1), and substituting $P_{j}=k_{j} P_{j+1}$ from (10), we get,

$$
K_{\mathrm{j}}=D_{j+1} / D_{j}
$$

Equation (19) is an important result for evaluating the final expression for CIR.

\section{B. Exact Closed-Form CIR Expressions for Two-Region Medium}

The formulation in the previous subsection will be used to obtain exact, closed-form expressions for the channel impulse response (CIR) of a two-region, MCvD channel with fully permeable interface boundaries. The fully permeable interfaces between regions allow all molecules to pass through the interface without any physical or chemical reactions and thus the permeability coefficient value is very large e.g., it goes to infinity. These exact closed-form expressions will be used as a benchmark to compare, verify and validate the simulations and other approximations in addition to computation of various system performance metrics. Referring to Fig.1, we assume that the number of regions ' $\mathrm{N}$ ' is equal to two and the region-1 $\left(\Omega_{1}\right)$ 
is defined in the region $z \geq z_{1}$ with diffusion coefficient $\mathrm{D}_{1}$ and concentration $\mathrm{P}_{1}$ while the region- $2\left(\Omega_{2}\right)$ is defined in the region $z<z_{1}$ with diffusion coefficient $\mathrm{D}_{2}$ and concentration $\mathrm{P}_{2}$.

Now, the concentrations in region-1 and region-2 in Laplace domain can be evaluated by substituting (13) and (17) in (12) as follows:

$$
\begin{aligned}
& \breve{P}_{1}=\int_{0}^{\infty}\left[\frac{e^{-s t_{0}}}{4 \pi D_{1}} \frac{1}{g_{1}} e^{-g_{1}\left|z-z_{0}\right|}+A_{1} e^{-g_{1} z}+B_{1} e^{g_{1} z}\right] J_{0}(\xi R) \xi d \xi \\
& \breve{P}_{2}=\int_{0}^{\infty}\left[A_{2} e^{-g_{2} z}+B_{2} e^{g_{2} z}\right] J_{0}(\xi R) \xi d \xi
\end{aligned}
$$

where the variables $(\xi, s)$ are omitted just to simplify the notation.

Applying the boundary conditions (6)-(9) for (20)-(21) and following the procedure indicated in Appendix B, we get the following weighting functions for a partially permeable interface:

$$
\begin{gathered}
A_{2}=B_{1}=0 \\
A_{1}=\frac{k_{1} \sigma_{1} D_{1} g_{1}-D_{2} g_{2}\left(\sigma_{1}-D_{1} g_{1}\right)}{4 \pi D_{1} g_{1}\left[k_{1} \sigma_{1} D_{1} g_{1}+D_{2} g_{2}\left(\sigma_{1}+D_{1} g_{1}\right)\right]} e^{g_{1}\left(2 z_{1}-z_{0}\right)} e^{-s t_{0}} \\
B_{2}=\frac{2 \sigma_{1}}{4 \pi\left[k_{1} \sigma_{1} D_{1} g_{1}+D_{2} g_{2}\left(\sigma_{1}+D_{1} g_{1}\right)\right]} e^{g_{1}\left(z_{1}-z_{0}\right)-g_{2} z_{1}} e^{-s t_{0}}
\end{gathered}
$$

For a fully permeable interface $\left(\sigma_{1} \rightarrow \infty\right),(23)$ and (24) reduces to following:

$$
\begin{aligned}
& A_{1}=\frac{k_{1} D_{1} g_{1}-D_{2} g_{2}}{4 \pi D_{1} g_{1}\left[k_{1} D_{1} g_{1}+D_{2} g_{2}\right]} e^{g_{1}\left(2 z_{1}-z_{0}\right)} e^{-s t_{0}} \\
& B_{2}=\frac{1}{2 \pi\left[k_{1} D_{1} g_{1}+D_{2} g_{2}\right]} e^{g_{1}\left(z_{1}-z_{0}\right)-g_{2} z_{1}} e^{-s t_{0}}
\end{aligned}
$$

Now, the expression for concentration in region-1 $\left(\Omega_{1}\right)$ with a fully permeable interface can be evaluated by substituting (22) and (25) in (20) given by

$$
\begin{aligned}
\breve{P}_{1}= & \underbrace{\frac{e^{-s t_{0}}}{4 \pi D_{1}} \int_{0}^{\infty} \frac{1}{g_{1}} e^{-g_{1}\left|z-z_{0}\right|} J_{0}(\mathrm{R} \xi) \xi d \xi}_{\breve{f}_{1}} \\
& +{\breve{f_{2}}}^{-\frac{e^{-s t_{0}}}{4 \pi D_{1}} \int_{0}^{\infty} \frac{1}{g_{1}} e^{-g_{1}\left(z_{0}+z-2 z_{1}\right)} J_{0}(\xi \mathrm{R}) \xi d \xi} \\
& +\underbrace{\frac{2 k_{1} e^{-s t_{0}}}{4 \pi} \int_{0}^{\infty} \frac{1}{\left[k_{1} D_{1} g_{1}+D_{2} g_{2}\right]} e^{-g_{1}\left(z_{0}+z-2 z_{1}\right)} J_{0}(\xi \mathrm{R}) \xi d \xi}_{\breve{f}_{3}}
\end{aligned}
$$

The terms $\breve{f}_{1}$ and $\breve{f}_{2}$ in (27) are evaluated in the time domain using [28, eq. (29.3.84)] along with the time and complex shifting properties of Laplace transform. Then, using [28, eq. (11.4.29)] and substituting $v=0$, we get

$$
\begin{aligned}
& f_{1}=\frac{1}{\left(4 \pi D_{1}\left(t-t_{0}\right)\right)^{3 / 2}} \exp \left(-\frac{R^{2}+\left(z-z_{0}\right)^{2}}{4 D_{1}\left(t-t_{0}\right)}\right), \quad t \geq t_{0} \\
& f_{2}=-\frac{1}{\left(4 \pi D_{1}\left(t-t_{0}\right)\right)^{3 / 2}} \exp \left(-\frac{R^{2}+\left(z_{0}+z-2 z_{1}\right)^{2}}{4 D_{1}\left(t-t_{0}\right)}\right), \quad t \geq t_{0}
\end{aligned}
$$

Substituting $g_{1}$ and $g_{2}$ from (14)-(15), $\breve{f}_{3}$ in (27) can be expressed using [30, eq. (3.310)] as $\breve{f}_{3}=\frac{k_{1} e^{-s t_{0}}}{2 \pi} \int_{0}^{\infty} \int_{0}^{\infty} \exp \left(-\left(\xi^{2}+\frac{s}{D_{1}}\right)^{\frac{1}{2}}\left[m k_{1} D_{1}+z_{0}+z-\right.\right.$

$\left.\left.2 z_{1}\right]\right) \times \exp \left(-\left(\xi^{2}+\frac{s}{D_{2}}\right)^{1 / 2} \mathrm{~m} D_{2}\right) \times J_{0}(\xi \mathrm{R}) \xi d \xi d m$

The inverse Laplace transform of (30) is evaluated in Appendix $\mathrm{C}$ and the final expression is given below:

$f_{3}=\int_{0}^{1} \frac{\frac{k_{1}}{8 \pi^{2}\left(D_{2} t\right)^{3 / 2}} \exp \left(-\frac{\alpha^{2} \grave{R}^{2}}{4\left(\left(1-v-\grave{t}_{0}\right)+\alpha^{2}\left(v-\grave{t}_{0}\right)\right)}\right)}{\left(v-\grave{t}_{0}\right)^{\frac{1}{2}}\left(1-v-\grave{t}_{0}\right)^{\frac{1}{2}}\left(\left(1-v-\grave{t}_{0}\right)+\alpha^{2}\left(v-\grave{t}_{0}\right)\right)} \mathcal{P}_{1}(v) d v$

where,

$$
\begin{aligned}
\mathcal{P}_{1}(v) & =\frac{-\left(\grave{z}_{0}+\grave{z}\right) v}{V^{2}} e^{-\frac{\left(\grave{z}_{0}+\grave{z}\right)^{2}}{4 v}}+\frac{\alpha k_{1}(\pi(1-v) v)^{1 / 2}}{V^{3 / 2}} \exp \left(\frac{-\left(\grave{z}_{0}+\grave{z}\right)^{2}}{4 V}\right) \\
& \times\left[1-\frac{\left(\grave{z}_{0}+\grave{z}\right)^{2}}{2 V}\right] \operatorname{erfc}\left(\frac{-k_{1} \alpha\left(\grave{z}_{0}+\grave{z}\right)(1-v)^{1 / 2}}{2 v^{1 / 2} V^{1 / 2}}\right)
\end{aligned}
$$

where, $\operatorname{erfc}($.$) denotes the complementary error function and$ $V=k_{1}^{2} \alpha^{2}(1-v)+v$.

The dimensionless parameters are defined below:

$$
\grave{z}_{0}=\frac{z_{1}-z_{0}}{\sqrt{D_{1} t}}, \grave{z}=\frac{z_{1}-z}{\sqrt{D_{1} t}}, \grave{R}=\frac{R}{\sqrt{D_{1} t}}, \alpha=\sqrt{\frac{D_{1}}{D_{2}}}, \grave{t}_{0}=\frac{t_{0}}{t}
$$

Assuming that the $\mathrm{TN}$ emits the information molecules initially at time $t_{0}=0$, the molecular concentration in the region$1\left(\Omega_{1}\right)$ can be obtained by summing of (28), (29), and (31) given by

$$
\begin{aligned}
P_{1} & =\frac{\sinh \left(\frac{\grave{z}_{0} \grave{z}}{2}\right)}{4\left(\pi D_{1} t\right)^{\frac{3}{2}}} \exp \left(-\frac{\grave{R}^{2}+\grave{z}_{0}^{2}+\grave{z}^{2}}{4}\right) \\
& +\frac{k_{1}}{8 \pi^{2}\left(D_{2} t\right)^{3 / 2}} \int_{0}^{1} \frac{\exp \left(-\frac{\alpha^{2} \grave{R}^{2}}{4\left(1-v+\alpha^{2} v\right)}\right)}{v^{1 / 2}(1-v)^{1 / 2}\left(1-v+\alpha^{2} v\right)} \mathcal{P}_{1}(v) d v
\end{aligned}
$$

where, $\sinh ($.$) is the hyperbolic sine function and \mathcal{P}_{1}(v)$ is given in (32).

The concentration for region-2 $\left(\Omega_{2}\right)$ with a fully permeable interface can be evaluated in a similar way by substituting (22) and (26) in (21) to get

$\breve{P}_{2}=\int_{0}^{\infty} \frac{e^{-s t_{0}}}{2 \pi\left(k_{1} D_{1} g_{1}+D_{2} g_{2}\right)} e^{g_{1}\left(z_{1}-z_{0}\right)-g_{2} z_{1}+g_{2} z} J_{0}(\xi R) \xi d \xi$

The expression for molecular concentration in region-2 $\left(\Omega_{2}\right)$ can also be expressed as

$$
P_{2}=\frac{1}{8 \pi^{2}\left(t D_{2}\right)^{3 / 2}} \int_{0}^{1} \frac{\exp \left(-\frac{\alpha^{2} \grave{R}^{2}}{4\left(1-v+\alpha^{2} v\right)}\right)}{v^{1 / 2}(1-v)^{1 / 2}\left(1-v+\alpha^{2} v\right)} \mathcal{P}_{2}(v) d v
$$

where,

$$
\begin{aligned}
\mathcal{P}_{2}(v)= & \frac{k_{1}^{3} \alpha^{4}(1-v) \grave{z}-v \grave{z}_{0}}{V^{2}} \exp \left(-\frac{\grave{z}_{0}^{2}(1-v)+\alpha^{2} \grave{z}^{2} v}{4 v(1-v)}\right) \\
& +\frac{k_{1} \alpha(\pi v(1-v))^{1 / 2}}{V^{3 / 2}} \exp \left(-\frac{\left(\alpha^{2} k_{1} \grave{z}+\grave{z}_{0}\right)^{2}}{4 V}\right) \\
& \times\left[1-\frac{\left(\alpha^{2} k_{1} \grave{z}+\grave{z}_{0}\right)^{2}}{2 V}\right] \operatorname{erfc}\left(\frac{\alpha\left(\grave{z} v-k_{1} \grave{z}_{0}(1-v)\right)}{2 v^{1 / 2}(1-v)^{1 / 2} V^{1 / 2}}\right)
\end{aligned}
$$

The finite integrals appearing in (34) and (36) can be easily evaluated numerically. 
It is important to note that we have verified that the CIR for the medium with two regions gets reduced to that of an unbounded single homogeneous medium given by (4), when $D=D_{1}=D_{2}, g=g_{1}=g_{2}$, and $k_{1}=1$ are substituted in (25)-(26) to evaluate the inverse Laplace transform of (20)-(21).

If the number of regions exceed two, i.e., for $\mathrm{N}>2$, it is difficult to evaluate the inverse Laplace transform in exact closed form and therefore require numerical approximations [40]. This will be discussed in the next subsection.

\section{C.CIR for Composite Medium with Multiple Regions $(N>2)$}

In this subsection, we will derive a closed-form expression for CIR in a diffusive medium with multiple-regions that is $\mathrm{N}>2$. As mentioned earlier, in this case we cannot invert Laplace transform in exact closed form and hence we resort to the use of Zakian's method for numerical inversion of Laplace transform [40]. After substituting (13) and (17) in (12), the general expression for molecular concentration (12) in Laplace domain for any region can be rewritten as

$\breve{P}_{i}=\int_{0}^{\infty}\left[\delta_{i 1} \frac{e^{-s t_{0}}}{4 \pi g_{i} D_{i}} e^{-g_{i}\left|z-z_{0}\right|}+A_{i} e^{-g_{i} z}+B_{i} e^{g_{i} z}\right] J_{0}(\xi R) \xi d \xi(38)$

where, $\delta_{i 1}$ is the Kronecker Delta function equal to one when $i=1$ and zero otherwise.

Applying the boundary conditions (6)-(9) for (38), we get

$$
\begin{gathered}
A_{N}=B_{1}=0 \\
\frac{D_{j}}{g_{j+1} D_{j+1}}\left[\frac{\delta_{j 1} e^{-s t_{0}}}{4 \pi D_{j}} e^{-g_{j}\left(z_{j}-z_{0}\right)}-g_{j}\left(A_{j} e^{-g_{j} z_{j}}-B_{j} e^{g_{j} z_{j}}\right)\right] \\
=B_{j+1} e^{g_{j+1} z_{j}}-A_{j+1} e^{-g_{j+1} z_{j}} \\
\delta_{j 1} \frac{e^{g_{j}\left(z_{j}-z_{0}\right)} e^{-s t_{0}}}{4 \pi g_{j} D_{j} k_{j}}+\frac{A_{j}}{k_{j}} e^{-g_{j} z_{j}}+\frac{B_{j}}{k_{j}} e^{g_{j} z_{j}} \\
=A_{j+1} e^{-g_{j+1} z_{j+1}}+B_{j+1} e^{g_{j+1} z_{j+1}}
\end{gathered}
$$

In general, the functions $A_{j}$ and $B_{j}$ can be obtained by solving the system of $2 \mathrm{~N}$ equations (40)-(41) for $j=1,2, \ldots, N$ using mathematical software packages such as Matlab or Mathematica. Therefore, the complexity of derivation an expression for the concentration will increase with the increase in the number of regions $\mathrm{N}$, unlike the simplistic averaging scenario considered in [19]-[20].

The inverse Laplace transform of the concentration given by (38) for any region can be evaluated using numerical inversion of Laplace Transform through Zakian's method as [40]

$$
P_{i}=\frac{2}{t} \sum_{m=1}^{5} \operatorname{Re}\left\{K(m) \breve{P}_{i}(s)\right\}
$$

where, $s=a(m) / t$ and the complex constants, $K(m)$ and $a(m)$, are listed in Table 9.2 in [40]. The expression (42) gives good numerical accuracy in the evaluation of the inverse Laplace transform, which can be evaluated numerically using Matlab.
The concentration expression (42) will be used for evaluating CIR for composite diffusive media having any number of regions by substituting (42) in (18). The results in section IV show that the expressions predict accurate results and matches well with the simulation results in spite of numerical approximations made during the evaluation of inverse Laplace Transform.

\section{COMMUNICATION METRICS}

Here, we discuss the procedure for obtaining the important communication metrics for a MCvD channel that may have either single or multiple regions. The communication metrics, viz., the pulse peak time, pulse peak amplitude and pulse width for an unbounded homogeneous MCvD channel with impulsive $\mathrm{TN}$ and passive $\mathrm{RN}$ are already available in the literature [11]. The pulse peak amplitude $\left(N_{\text {peak }}\right)$ varies over separation distance, which may be interpreted as the channel attenuation. This has significant impact on the detection of molecular signals. Furthermore, the pulse peak time $\left(t_{\text {peak }}\right)$ determines the communication delay between the transmission and reception of molecular signals. The pulse peak amplitude for a homogeneous unbounded MCvD channel does not depend on the diffusion coefficient $\mathrm{D}$ while the pulse peak time is inversely proportional to it [11]. This suggests that any increase in the diffusion coefficient results in a corresponding increase in the speed at which molecules diffuse, thus, causing a corresponding decrease in the pulse peak time. Another important metric is the pulse width, which mainly affects the achievable transmission rate in a molecular communication system. Similar to EM communications, the pulse width of a received signal is defined as the time duration at which the signal level is greater than its half-maximum value (i.e., $1 / 2$ $N_{\text {peak }}$ ). For RN to correctly decode the molecular pulse data emitted by $\mathrm{TN}$, the separation between the transmitted pulses need to be approximately equal to the pulse width, assuming perfect synchronization. Thus, the transmission rate can be approximately expressed as the inverse of the pulse width [11].

However, finding the communication metrics in closed form for a diffusive medium consisting of two or more regions require analytical time derivatives of the concentration e.g., (34), (36), or (42), which could lead to additional mathematical complexities. To avoid this, in this paper, we numerically find the maximum value (peak amplitude) of (18) for both (34) and (36) for a medium with two-regions and record the corresponding time instant (peak time) for various TN-RN separations. Furthermore, the pulse width for region-1 and region-2 can be numerically evaluated by finding both their amplitudes and the corresponding time instants at which the concentration calculated using (34) and (36) exceed values greater than the half-maximum value. The pulse width is then equal to the difference between these two time instants. Through this, we establish that, the pulse peak amplitude depends on the diffusion coefficients, which will be further discussed in the next section. 


\section{ANALYTICAL AND SIMULATION RESULTS}

In this section, verification and validation of the simulations with the analytical results calculated from Sections II-III are carried out. The simulation results are averaged over number of independent realizations and in general, we observe a perfect match between the simulation and the analytical results. The parameters considered for the simulation are listed in Table-I.

TABLE I

THE SIMULATION PARAMETERS (UNLESS STATED OTHERWISE)

\begin{tabular}{ccl}
\hline \hline Parameter & Value & \multicolumn{1}{c}{ Description } \\
\hline $\mathrm{Q}$ & $10^{5} \mathrm{~mol}$. & No. of released molecules by TN. \\
$T_{a}$ & $330 \mathrm{~K}$ & Absolute medium temperature. \\
$\eta_{\text {Water }}$ & $0.6913 \mathrm{~g} / \mathrm{m} . \mathrm{s}$ & Water viscosity [31]. \\
$\eta_{\text {Blood }}$ & $2.46 \mathrm{~g} / \mathrm{m} . \mathrm{s}$ & Blood viscosity [32]. \\
$\eta_{\text {Air }}$ & $18.27 \times 10^{-3} \mathrm{~g} / \mathrm{m} . \mathrm{s}$ & Air viscosity [32]. \\
$D_{\text {water }}$ & $131.3 \mu \mathrm{m}^{2} / \mathrm{s}$ & Water diffusion coefficient using (2) \\
$D_{\text {Blood }}$ & $36.9 \mu \mathrm{m}^{2} / \mathrm{s}$ & Blood diffusion coefficient using (2) \\
$D_{\text {Air }}$ & $5000 \mu \mathrm{m}^{2} / \mathrm{s}$ & Air diffusion coefficient using (2). \\
$r_{m}$ & $2.5 \mathrm{~nm}$ & Radius of insulin molecule [33]. \\
$R_{r x}$ & $2 \mu \mathrm{m}$ & Radius of RN. \\
$\left(x_{0}, y_{0}, z_{0}\right)$ & $(5,5,10) \mu \mathrm{m}$ & Coordinate of TN center. \\
$(x, y, z)_{1}$ & $(1,1,25) \mu \mathrm{m}$ & Coordinate of RN center in region 1. \\
$(x, y, z)_{2}$ & $(1,1,-5) \mu \mathrm{m}$ & Coordinate of RN center in region 2. \\
$d$ & $5-30 \mu \mathrm{m}$ & Distance between TN and RN. \\
$\left(Z_{\text {sep }}, Z_{\text {sep }}^{\prime}\right)$ & $1-10 \mu \mathrm{m}$ & Separation from the interface to TN \\
$\Delta t$ & & and RN along z-axis, respectively. \\
$\Delta t$ & $0.1 \mathrm{~ms}$ & Simulation time step. \\
$T_{s}$ & $4 \mathrm{~s}$ & Total simulation time. \\
$I$ & 100 & Number of realizations. \\
\hline \hline
\end{tabular}

We also propose a comprehensive framework for particlebased simulation of 3-D diffusion in a composite, molecular communication channel with distinct multiple regions. These simulations, which are developed using MATLAB, will be validated for a two, three, and four region $\mathrm{MCvD}$ channel using the analytical CIR derived in the section II. In the proposed simulator, the simulation time $T_{s}$ is divided into $N_{t}$ time steps with $\Delta t$ step width. The emission of molecules by the TN at the beginning of simulation time and at an initial location $\left(\mathrm{x}_{0}, \mathrm{y}_{0}\right.$, $\mathrm{z}_{0}$ ) is modelled using an impulse function. Then, the molecules are made to move independently of each other, in all directions following Brownian motion. The coordinates of the molecule $\left(x_{i}, y_{i}, z_{i}\right)$ at the time $t=i \Delta t$ are updated using [34]

$$
\left(x_{i}, y_{i}, z_{i}\right)=\left(x_{i-1}, y_{i-1}, z_{i-1}\right)+\left(\Delta x_{i}, \Delta y_{i}, \Delta z_{i}\right)
$$

where $i=1, \ldots, N_{t}, N_{t}$ is the total number of simulation time steps, $\left(x_{i-1}, y_{i-1}, z_{i-1}\right)$ are the coordinates of the molecule at the time $t=(i-1) \Delta t,\left(\Delta x_{i}, \Delta y_{i}, \Delta z_{i}\right) \sim N\left(0, \sigma^{2}\right)$ are the random displacements over each spatial axis during $\Delta \mathrm{t}$ which follows the normal distribution with zero-mean and variance $\sigma^{2}=2 D_{j} \Delta t$ for $j=1$, $2, \ldots, \mathrm{N}$.

When a molecule moves from its current position in the $\mathrm{j}^{\text {th }}$ region to a new position in the $(j \pm 1)^{\text {th }}$ region across the boundary interface, its displacement will be impacted by the diffusion coefficient of the new $(j \pm 1)^{\text {th }}$ region. If it falls within the RN's sensing range, it will be counted without absorption and the RN counter will be increased by one at the end of time step. The RN counter value at each time step represents the total number of received molecules at that time. Here, it is assumed that $\mathrm{RN}$ has the capability of counting the number of received molecules during a time slot [35]. Once the received CIR is obtained from the simulation, the communication metrics are evaluated following the procedure given in section III. In this paper, all simulation results are obtained by averaging over 100 independent realizations from which the samples are chosen for the plots.

The insulin hormone molecule is chosen as an information molecule [36], [37]. In the rest of the paper, air, water, and blood within the cells are assumed to be a separate distinct regions for computation and comparison. The transmitter (TN) is assumed to be always placed in region-1. Moreover, we compare our CIR results with those obtained using an effective diffusion coefficient at steady state as given in the literature [19]-[20] and demonstrate the latter's inability in examining the time dependent channel characteristics which may be only useful to measure the effective properties of diffusive media with multiple regions [21]. The following notation will be employed for a two-region medium: (i) "W-B" to indicate water in region- 1 and blood in region-2 and (ii) "B-W" to indicate the reversal of the above. Similar notation for a medium with threeregions is followed meaning " $\mathrm{A}-\mathrm{W}-\mathrm{B}$ " indicating "Air-WaterBlood" forming the three regions in that order respectively. We will also investigate the effect of the location of interface boundary with respect to $\mathrm{TN}\left(\mathrm{Z}_{\text {sep }}\right)$ and $\mathrm{RN}\left(\mathrm{Z}_{\text {sep }}\right)$, respectively and also the effects of the thickness of different regions. Note, to indicate the CIR for $i^{\text {th }}$ region, we just use $\mathrm{CIR}_{i}$, e.g., $\mathrm{CIR}_{2}$ for region-2.

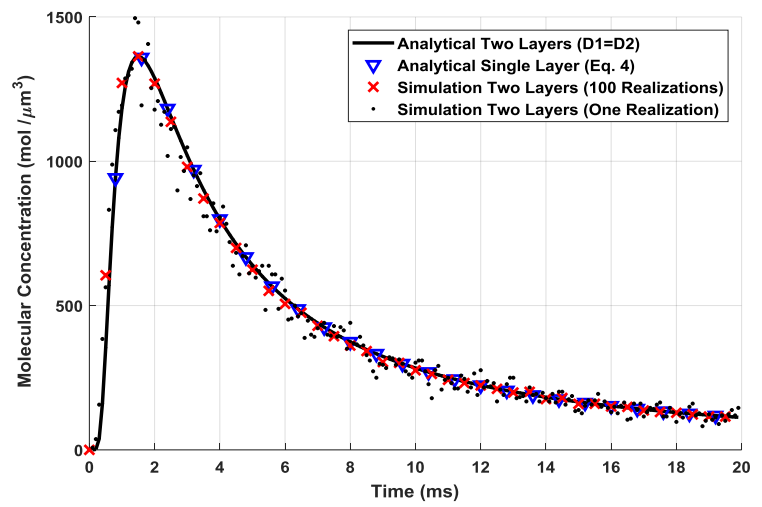

Fig. 2. Molecular concentration for two-region media with equal diffusion coefficients.

It is expected that the CIR derived for a MCvD medium with multiple regions should reduce to that of a single homogeneous medium, when the diffusion coefficients of all regions are made equal. To verify this, in the two-region analytical expressions, we make both the diffusion coefficients equal $\left(D_{1}=D_{2}=1 \times 10^{-9}\right.$ $\mathrm{m}^{2} / \mathrm{s}$ ) and obtained the molecular concentration as a function of time. These are compared then, with analytical results obtained for a single region with $\mathrm{D}=1 \times 10^{-9} \mathrm{~m}^{2} / \mathrm{s}$ along with simulations as shown in Fig.2. For this, the TN-RN separation distance is considered to be $3 \mu \mathrm{m}$, the number of released molecules is $Q=5 \times 10^{5}$, and the radius of $\mathrm{RN}$ is $0.4 \mu \mathrm{m}$ [11]. The simulation results obtained for a single realization are also compared with results that are averaged over 100 independent realizations in Fig.2. 
The CIR in region-2 $\left(\mathrm{CIR}_{2}\right)$ for a medium with two-regions is computed assuming that the $\mathrm{RN}$ is placed in region-2 using (18) and (36). The $\mathrm{CIR}_{2}$ is evaluated for both 'W-B' and 'B-W' cases and by varying interface separations $Z_{\text {sep }}$ and $Z_{\text {sep }}$ with respect to $\mathrm{TN}$ and $\mathrm{RN}$, respectively. For computing the tworegion results shown in Fig. 3, we assume constant TN-RN separation, the TN is placed in region- 1 and $\mathrm{RN}$ in region- 2 . The CIR results are plotted in Fig. 3(a) and (b) along with those obtained from particle-based simulation. It can be observed from the plots that the $\mathrm{CIR}_{2}$ largely depends on $Z_{\text {sep }}$ and $Z_{\text {sep. }}$. For W-B medium, with increasing $Z_{\text {sep }}$, the pulse peak amplitude of $\mathrm{CIR}_{2}$ increases while the pulse peak time and pulse width decrease. However, the trend appears to be reversed with increasing $Z_{\text {sep }}^{\prime}$. This may be due to increase in the thickness of the water region between $\mathrm{TN}$ and the interface, which has higher diffusion coefficient than blood. Thus, as $Z_{\text {sep }}$ increases, the probability of the molecules to diffuse faster from region-1 (Water) towards region-2 (Blood) increases, and as a result, larger numbers of molecules reach the $\mathrm{RN}$ faster to increase the peak amplitude but with corresponding reduction in the peak time and pulse width. When the regions are reversed, i.e., for the B-W case, as expected, the trend reverses since the thickness of blood region that lies between $\mathrm{TN}$ and the interface increases. In addition, blood's diffusion coefficient is lower than that of water.

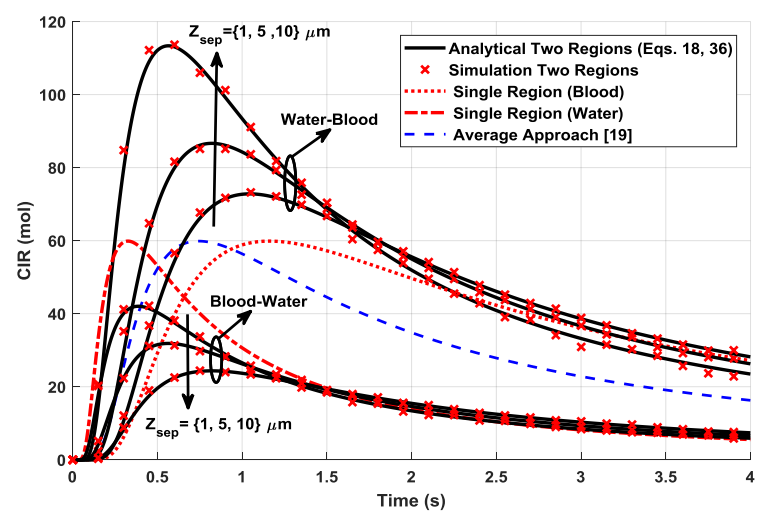

(a)

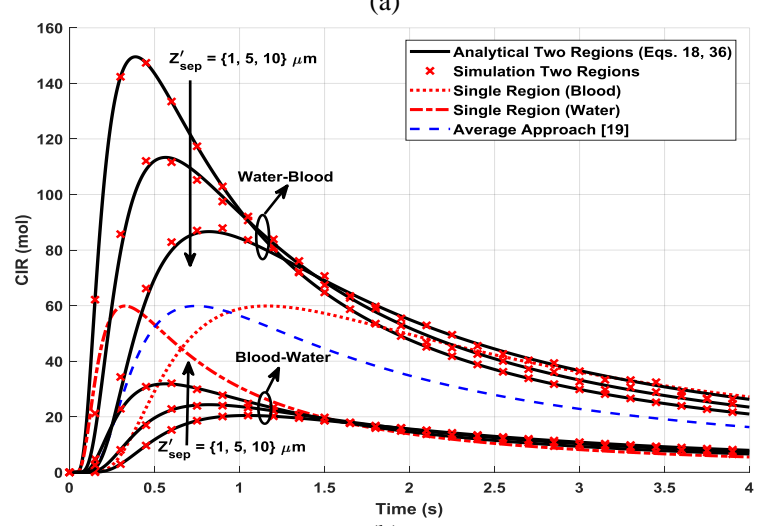

(b)

Fig. 3. The $\mathrm{CIR}_{2}$ as a function of time in a two-region medium for (a) different distances between $\mathrm{TN}$ and the interface $\left(\mathrm{Z}_{\mathrm{sep}}\right)(\mathrm{b})$ different distances between $\mathrm{RN}$ and the interface $\left(\mathrm{Z}_{\mathrm{sep}}\right)$
We now demonstrate using comparisons as to why a CIR of a multiple-region MCvD channel cannot accurately be modeled by an averaged equivalent medium with averaged diffusion coefficient as suggested in [19]-[20]. In Fig.3, these results are compared with those obtained by the average diffusion coefficient approach [19]-[20]. As shown in Fig. 3, for all interface separations $Z_{\text {sep }}$ and $Z_{\text {sep }}^{\prime}$, the CIR results obtained using averaging approach [19]-[20] deviate significantly from the actual CIR results of a two-region medium (both for W-B and B-W). This large deviation in CIR suggests that the averaging approach given in [19]-[20] cannot accurately model the CIR of a two-region MCvD channel. We find that it even holds true for a multi-region channel as will be discussed later.

For comparison sake, the CIR of a single region case is computed using diffusion coefficient of region-2 $\left(\mathrm{D}_{2}\right)$ where the $\mathrm{RN}$ is located and it is compared with the CIR of a two-region medium. As shown in Fig. 3, a larger mismatch in results can be seen when the TN in region-1 is moved far away from the interface (e.g., $Z_{\text {sep }}=10 \mu \mathrm{m}$ ) but, it reduces when the TN moves closer to the interface (e.g., $Z_{\text {sep }}=1 \mu \mathrm{m}$ ). This may be due to decrease thickness of region between $\mathrm{TN}$ and the interface as the TN moves closer to the interface, which in order makes most of the molecules to move to region-2 and diffusing most of time in region-2 where the $\mathrm{RN}$ is located. Thus, the diffusion coefficient of region-2 plays a dominant role in this case. However, when the $\mathrm{RN}$ in region- 2 is moved far from the interface (e.g., $Z_{\text {sep }}^{\prime}=10 \mu \mathrm{m}$ ), initially, the mismatch appears to be smaller but as RN gets closer to the interface, it eventually has increased. This due to fact that, when the RN moves closer to the interface, it appears that the molecules that contribute to $\mathrm{CIR}_{2}$ will mainly be influenced by the diffusion coeffcent of region-1 $\left(D_{1}\right)$ most of the time because the thickness of region between $\mathrm{RN}$ and the interface decreases.

It can be seen in Fig. 3 that both approaches, i.e., the CIR of averaging approach [19] and the CIR of a single homogeneous region, result in the same pulse peak amplitude since, for a single homogeneous medium, the peak amplitude of CIR does not depend on the diffusion coefficient.

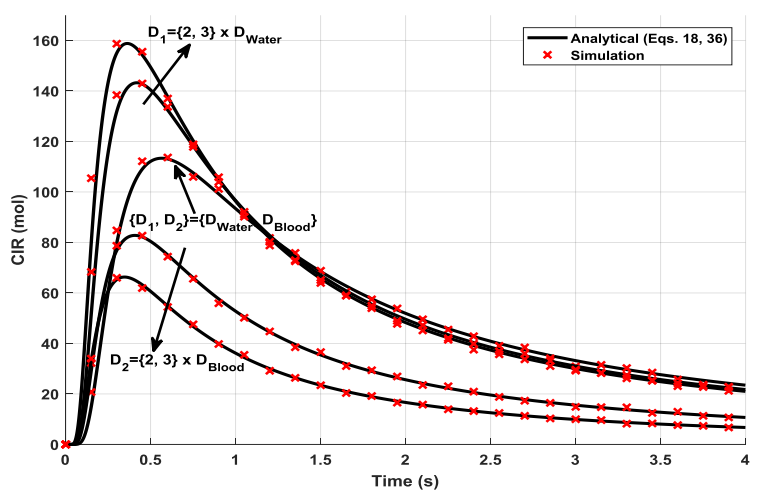

Fig. 4. The time dependence of $\mathrm{CIR}_{2}$ for region-2 when diffusion coefficients $\left(D_{1}, D_{2}\right)$ are varied in integer multiples of $D_{\text {Water }}$ and $D_{\text {Blood }}$ for $Z_{\text {sep }}=10 \mu \mathrm{m}$.

Fig.4 illustrates how the diffusion coefficient of each region influences the temporal behaviour of $\mathrm{CIR}_{2}$ when the diffusion 
coefficients are varied in integer multiples $(\mathrm{m}=1,2,3)$ of water and blood viz., $\mathrm{D}_{1}=\mathrm{m} \times \mathrm{D}_{\text {Water }}$ and $\mathrm{D}_{2}=\mathrm{m} \times \mathrm{D}_{\text {Blood. }}$. When the $\mathrm{RN}$ is located in region-2, one can observe from Fig. 4, that the diffusion coefficients influence the $\mathrm{CIR}_{2}$, peak amplitude, peak time, and pulse width significantly.

Fig. 5(a) shows the pulse peak amplitude of $\mathrm{CIR}_{2}$ versus TN$R N$ separation for $D_{1}=m \times D_{\text {Blood }}$ and $D_{2}=m \times D_{\text {Water }}$ where $m=1$, 2 is an integer multiple. Here, we assume fixed TN-RN separation, the TN is placed in region- 1 and $\mathrm{RN}$ in region-2. An increase in $\mathrm{D}_{1}$ increases the probability for the molecules to diffuse faster across the interface from region-1 into region-2, which causes the pulse peak amplitude to increase at the $\mathrm{RN}$ in region-2. The peak amplitude of $\mathrm{CIR}_{2}$ decreases with increase in $\mathrm{D}_{2}$, because it increases the probability for the molecules to return back from region-2 to region-1 which contribute to reduction of the pulse peak amplitude at $\mathrm{RN}$ in region-2. More importantly, these results establish that in a two region diffusive medium, the pulse peak amplitude is highly dependent on the diffusion coefficients unlike in a single region medium where it is independent of the diffusion coefficient [11]. Consider a specific example where the $R N$ is located at distance $d=14 \mu \mathrm{m}$ from the TN that is positioned near the interface with $Z_{\text {sep }}=1 \mu \mathrm{m}$. The CIR peak amplitude for this case, as in Fig. 5(a), shows an increase by a factor of 1.2 when $\mathrm{D}_{1}=2 \times \mathrm{D}_{\text {Blood }}$ but when $\mathrm{D}_{2}=2 \times \mathrm{D}_{\text {Water, }}$, it decreases by a factor of 0.7 . However, when the $\mathrm{TN}$ is placed far from the interface i.e., for $Z_{\mathrm{sep}}=10 \mu \mathrm{m}$, the pulse peak amplitude increases by a factor of 1.8 for $\mathrm{D}_{1}=2 \times \mathrm{D}_{\text {Blood }}$ but it decreases by a factor of 0.4 for $\mathrm{D}_{2}=2 \times \mathrm{D}_{\text {Water. }}$. It can also be seen that the pulse peak amplitude decreases with the increasing separation between $\mathrm{TN}$ and $\mathrm{RN}$ since only a few molecules can reach the RN. For "B-W" medium, when the TN is placed near the interface $\left(Z_{\text {sep }}=1 \mu \mathrm{m}\right)$, doubling the TN-RN separation reduces the pulse peak amplitude approximately by a factor of $1 / 8$ (i.e., $\mathrm{N}_{\text {peak }} \propto 1 / \mathrm{d}^{3}$ ).

Fig. 5(b) shows the pulse peak time of $\mathrm{CIR}_{2}$ as a function of TN-RN separation when $\mathrm{D}_{1}$ and $\mathrm{D}_{2}$ are following $\mathrm{D}_{1}=\mathrm{m} \times \mathrm{D}_{\text {Water }}$ and $\mathrm{D}_{2}=\mathrm{m} \times \mathrm{D}_{\text {Blood }}(\mathrm{m}=1,2$ is an integer multiple). It can be observed that for any increase in either $D_{1}$ or $D_{2}$ or both, the peak time of $\mathrm{CIR}_{2}$ decreases. However, the effect of the diffusion coefficient $\mathrm{D}_{2}$ is dominant on the peak time of $\mathrm{CIR}_{2}$ since the RN is located in region-2. Thus, we can see that the pulse peak time is mainly influenced by $\mathrm{D}_{2}$. As $\mathrm{D}_{2}$ increases, the pulse peak time sharply decreases because the molecules in region-2 will diffuse faster to reach the $\mathrm{RN}$, which coincidentally, concur with the literature [11]. Results also indicate the influence of $\mathrm{D}_{1}$ on the peak time of $\mathrm{CIR}_{2}$ is smaller when the TN is placed closer the interface $\left(Z_{\text {sep }}=1 \mu \mathrm{m}\right)$. This may be due to the increased probability for the molecules released by TN to move into region-2 under the influence of diffusion coefficient of region-2, most of the time. For example, when the $\mathrm{RN}$ is separated at a distance $\mathrm{d}=28 \mu \mathrm{m}$ from the TN which is placed closer to the interface $\left(Z_{\text {sep }}=1 \mu \mathrm{m}\right)$, the pulse peak time decreases by a factor of 0.97 for $\mathrm{D}_{1}=2 \times \mathrm{D}_{\text {Water }}$ and by a factor of 0.5 for $D_{2}=2 \times D_{\text {Blood. }}$ On other the hand, when, the $\mathrm{TN}$ is placed far from the interface $\left(\mathrm{Z}_{\mathrm{sep}}=10 \mu \mathrm{m}\right)$, the pulse peak time decreases by a factor of 0.9 for $\mathrm{D}_{1}=2 \times \mathrm{D}_{\text {Water }}$ and by a factor of 0.6 for $\mathrm{D}_{2}=2 \times \mathrm{D}_{\text {Blood. }}$.

The pulse peak time also increases with the increase in the separation between TN and RN because it will take longer time for the molecules to reach the RN. For example, for the "W-B" medium, if the TN-RN separation is doubled, the pulse peak time is four times longer when the $\mathrm{TN}$ is placed near the interface $\left(Z_{\text {sep }}=1 \mu \mathrm{m}\right)$ and six times longer when the TN is placed far from the interface $\left(Z_{\mathrm{sep}}=10 \mu \mathrm{m}\right)$ as indicated in Fig. $5(b)$.

Fig. 5(c) illustrates the changes in CIR pulse width in region2 with TN-RN separation when the diffusion coefficients $D_{1}$ and $\mathrm{D}_{2}$ are varied in multiples of $\mathrm{D}_{\text {Water }}$ and $\mathrm{D}_{\text {Blood }}$, respectively. It can be seen from the plots that the pulse width follows similar trend as that of the pulse peak time. The separation between the transmitted pulses need to be approximately equal to the pulse width for $\mathrm{RN}$ to correctly decode the received molecular pulse data. Unlike the EM communication where the pulse width does not depend on the transmission distance, the pulse width in MC increases with the increase in the transmission distance resulting in lower data rates. As an example, if the transmission distance is doubled, the pulse width becomes approximately four times wider and correspondingly the transmission rate decreases by $25 \%$ as shown in Fig. 5(c). Furthermore, in a two region diffusive channel, our results suggest that the pulse width can be reduced to half (50\%) by doubling the diffusion coefficient $\mathrm{D}_{2}$ so that the transmission rate can be doubled.

The CIR for a medium with two regions when both TN and $\mathrm{RN}$ are placed in the same region, i.e., in region-1 (denoted as $\mathrm{CIR}_{1}$ ) can be obtained from (18) and (34). Fig. 6 (a) compares of $\mathrm{CIR}_{1}$ as function of time for varying interface separation distances $\left(Z_{\text {sep }}\right)$ from the TN. Since both TN and RN are located in the same region-1, the effect of both of their separations from the interface $\left(Z_{\text {sep }}^{\prime}, Z_{\text {sep }}\right)$ have the same dependency on $C_{1 R}$ and hence not plotted here. However, the peak amplitude increases with increase in either $Z_{\text {sep }}$ or $Z_{\text {sep }}$ for Water-Blood medium, while the trend gets reversed for Blood-Water medium. For WB medium, this may be due to the increased probability for molecules to diffuse faster mainly under the influence of the diffusion properties of region-1 (water). For B-W medium, the trend gets reversed because blood has lower diffusion coefficient than water.

These results are also compared with those of averaging approach presented in [19]-[20] for multiple region CIR to highlight the deficiencies of the latter. As expected, for both W$\mathrm{B}$ and $\mathrm{B}-\mathrm{W}$ (two region) media, the results obtained using the averaging approach show a large mismatch with our $\mathrm{CIR}_{1}$ results for all interface separations. The $\mathrm{CIR}_{1}$ results obtained by considering the medium to have two distinct regions do not match with the results obtained by considering the medium to be a single homogeneous medium. Particularly, this mismatch is seen to increase if the TN gets closer to the interface (e.g., $Z_{\text {sep }}=1 \mu \mathrm{m}$ ) while it decreases when the TN is placed far from the interface (e.g., $Z_{\text {sep }}=10 \mu \mathrm{m}$ ). The decrease in mismatch for 
larger separation of $\mathrm{TN}$ from the interface is due to increase in the thickness of region between $\mathrm{TN}$ and the interface and thus helping the molecules to diffuse most of the time in region-1. As a consequence, the effect of region- 2 almost disappears, making the two-region medium to behave as though it is an effective homogeneous single region with diffusion coefficient $\mathrm{D}_{1}$. But, when the TN gets closer to the interface, effective single region approximation cannot be employed anymore to obtain the $\mathrm{CIR}_{1}$ of a two-region medium because the molecules will mainly diffuse in region- 2 most of the time.

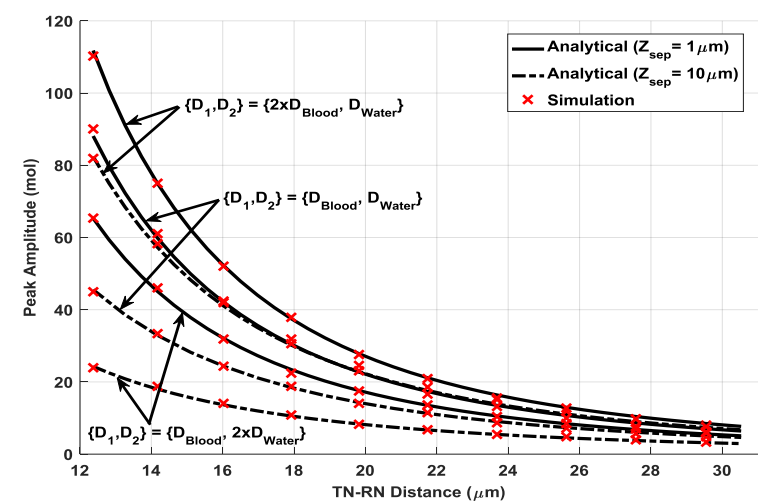

(a)

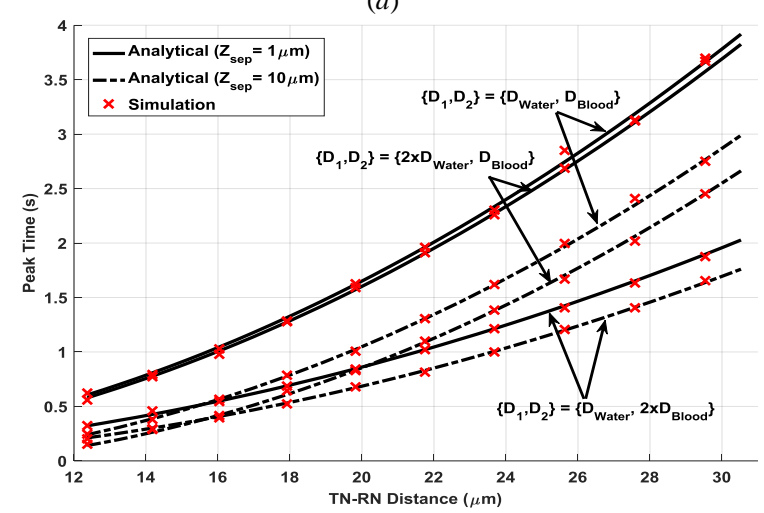

(b)

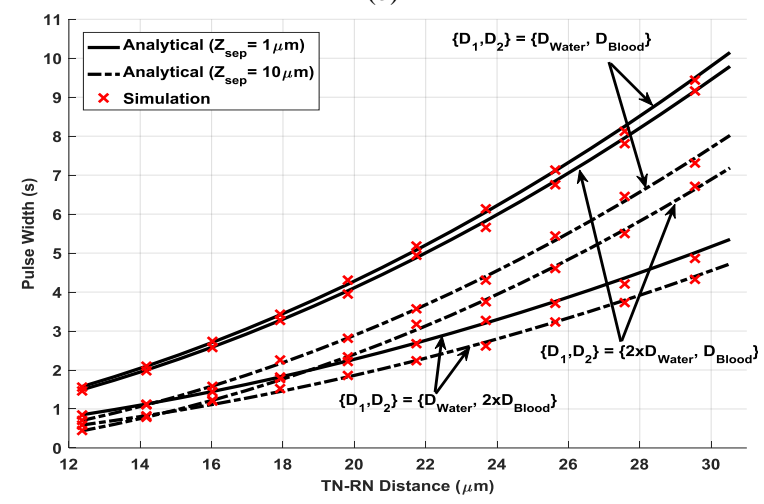

(c)

Fig. 5. The communication metrics of $\mathrm{CIR}_{2}$ (for region-2) versus $\mathrm{TN}-\mathrm{RN}$ separation and for different values of diffusion coefficient (a) the pulse peak amplitude (b) the pulse peak time (c) the pulse width.

Fig. 6 (b) shows the $\mathrm{CIR}_{1}$ of region-1 as a function of time when the diffusion coefficients of both the regions are increased in integer steps viz., $\mathrm{D}_{1}=\mathrm{m} \times \mathrm{D}_{\text {Blood }}$ and $\mathrm{D}_{2}=\mathrm{m} \times \mathrm{D}_{\text {Water }}$. The plots specify how the variations in diffusion coefficients affect the
$\mathrm{CIR}_{1}$ peak amplitude, peak time, and pulse width when the TN is placed far from the interface $\left(\mathrm{Z}_{\mathrm{sep}}=10 \mu \mathrm{m}\right)$. As shown in Fig. 6 (b), when the TN is placed far from the interface $\left(Z_{\text {sep }}=10 \mu \mathrm{m}\right)$, both the diffusion coefficients $\mathrm{D}_{1}$ and $\mathrm{D}_{2}$ have no impact on the peak amplitude of $\mathrm{CIR}_{1}$ since the medium acts effectively as a single region medium for which the pulse peak amplitude is independent of the diffusion coefficient. However, our results have indicated that when the $\mathrm{TN}$ is moved closer to the interface (e.g., $Z_{\text {sep }}=1 \mu \mathrm{m}$ ), both $\mathrm{D}_{1}$ and $\mathrm{D}_{2}$ have slight impact on the peak amplitude of $\mathrm{CIR}_{1}$. From Fig. 6 (b), one can observe that the peak time of $\mathrm{CIR}_{1}$ is pre-dominantly determined by the diffusion coefficient $\mathrm{D}_{1}$ since the $\mathrm{RN}$ is located in region-1. As $\mathrm{D}_{1}$ increases, the pulse peak time decreases because the molecules in region-1 will diffuse faster to reach the RN at region-1, which concurs with the literature [11]. Also, since the $\mathrm{RN}$ is located in region-1, the diffusion coefficient of region-2 $\left(D_{2}\right)$ will have negligible impact on the peak time of $\mathrm{CIR}_{1}$. The plots for the communication metrics of $\mathrm{CIR}_{1}$ in region-1 as a function of the separation distance are omitted here due to page length limitations but effect of the diffusion coefficients on these metrics can be observed from Fig. 6.

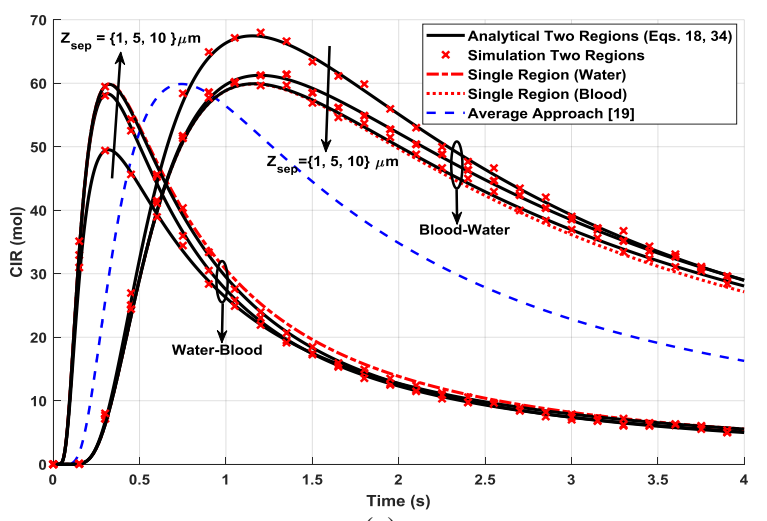

(a)

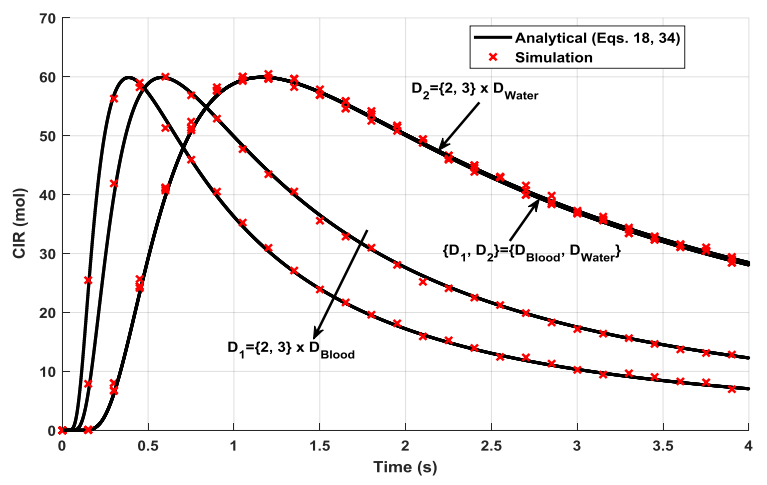

(b)

Fig. 6. The $\mathrm{CIR}_{1}$ of region-1 as a function of time for (a) varying separation distances between $\mathrm{TN}$ and the interface $\left(\mathrm{Z}_{\mathrm{sep}}\right)(\mathrm{b})$ varying diffusion coefficients (D1, D2).

Fig. 7 (a) shows the $\mathrm{CIR}_{3}$ as a function of time for a $\mathrm{MCvD}$ medium with three regions where the TN placed in region- 1 and the $\mathrm{RN}$ in region-3. In this figure, the CIR is evaluated using (18) and (42) for various values of the separation distance between the TN and the interface $\left(Z_{\text {sep }}\right)$. When the first, second, and third regions are formed with air, water, and blood respectively, the $\mathrm{CIR}_{3}$ has higher peak amplitude (and lower peak time) compared to the case when the second and third 
regions are swapped i.e., region- 2 becomes blood and region-3 becomes water. The reason for this occurrence may be due to the molecules moving faster in the region that has higher diffusion coefficient where in this case, the region having water has a higher diffusion coefficient than blood. For example, when the medium is Air-Water-Blood, the molecules will move faster in region-2 (water) and they will take shorter time to reach region-3 (blood) and thus most of the molecules will enter region-3 (blood) quickly and the number of molecules that reach the $\mathrm{RN}$ will increase but the peak time will decrease. Also in a three-region medium, as the separation distance between TN and the interface, $Z_{\text {sep }}$, between region-1 and region-2 increase, there is a corresponding increase of the peak amplitude but the peak time decreases for both the cases viz., Air-Blood-Water and Air-Water-Blood. This is because, the region-1, where the TN placed, is Air in both these cases which has higher diffusion coefficient than water and blood. Thus, as $Z_{\text {sep }}$ increases, the thickness of the region, which is filled by air between TN and the interface, increases which makes most of the molecules to move faster in region-1 (air) before they reach region-2. To confirm this, we perform a simulation for monitoring the number of the molecules as a function of time in each region as shown in Fig. 7 (b). We find that the number of the molecules in region-2 (water) reaching its peak value in a shorter time but decreases rapidly compared to when region2 is filled with blood. The reason for large reduction in the number of the molecules in region- 2 when it is filled with water is because the molecules that travel to region-3 where the RN located increase and it appears only few of them go back to region-1. The molecules stay only for shorter duration in region-2 (water) before they move to region-3, while for A-B$\mathrm{W}$ medium most of the molecules take longer time to diffuse in region-2 (blood) before reaching the region-3. This explains why the CIR changes as the regions are swapped. Moreover, as shown in Fig. 7 (a), the CIR using averaging approach [19] shows high mismatch with the exact CIR results for both A-W$\mathrm{B}$ and $\mathrm{A}-\mathrm{B}-\mathrm{W}$ mediums.

Now consider a CIR of a MCvD channel with four regions. The time dependency of the $\mathrm{CIR}_{4}$ on the thicknesses of region2 and region- 3 when the four regions are formed by Air-WaterBlood-Water and when the RN placed in region- 4 is shown in Fig. 8. The CIR results plotted in Fig. 8 are evaluated using (18) and (42) which indicate that the amplitude of CIR increases with corresponding decrease in the peak time as the thickness of region-2 (water) increases and thickness of region-3 (blood) decreases. This is because the water has higher diffusion coefficient than blood. Further, with the increase in the thickness of water region and decrease in the thickness of blood region, most of the molecules will be influence by the water region for majority of the time than the blood region. Thus, number of molecules that move to region-4 increases and they reach the $\mathrm{RN}$ in a shorter time as the thickness of the water region increase. This clearly highlights the deficiencies in the averaging approach suggested in [19], which fails to provide accurate predictions for a four-region medium.

Thus, for a passive receiver, which is located in $\mathrm{MCvD}$ medium having multiple regions, the results reveal that the diffusion coefficient has significant effect on the CIR, pulse peak amplitude, pulse peak time, and pulse width. On the other hand, when the medium is a single homogeneous region, the peak amplitude does not depend on the diffusion coefficient as predicted in [11]. In addition, the results show that thickness of each region and the separation distance between either the TN or RN with respect to the region interface has significant effect on the CIR. Thus, the CIR expressions derived in this paper as well as the proposed particle-based simulator are helpful for the design of optimum TN and RN nano machines, equalization schemes and detection techniques for MCvD systems in a threedimensional diffusive channel having multiple regions.

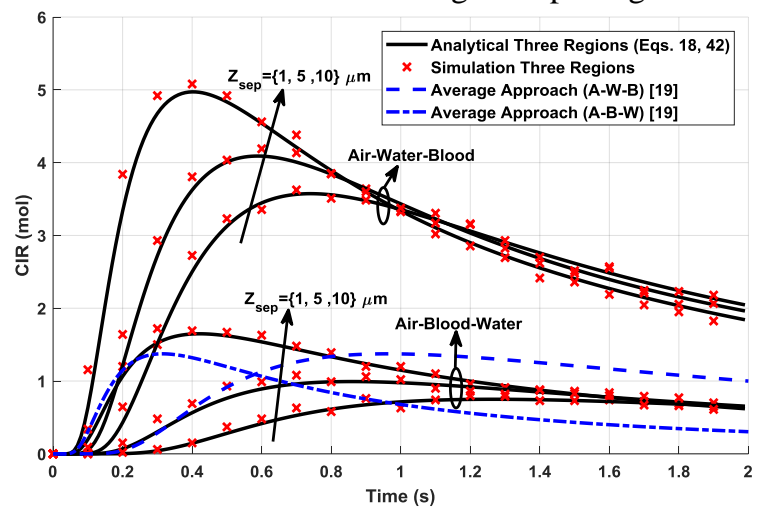

(a)

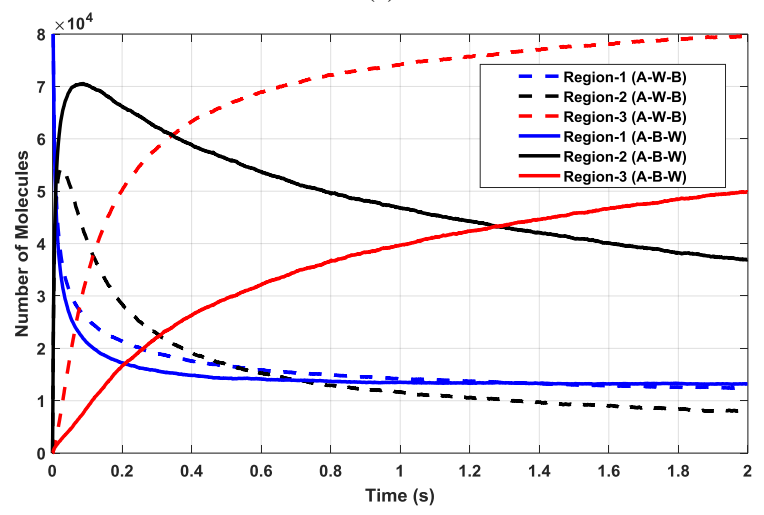

(b)

Fig. 7. (a) The CIR as a function of time for three-region medium with different separation $Z_{\text {sep }}$ (b) Total number of molecules in each region as a function of time

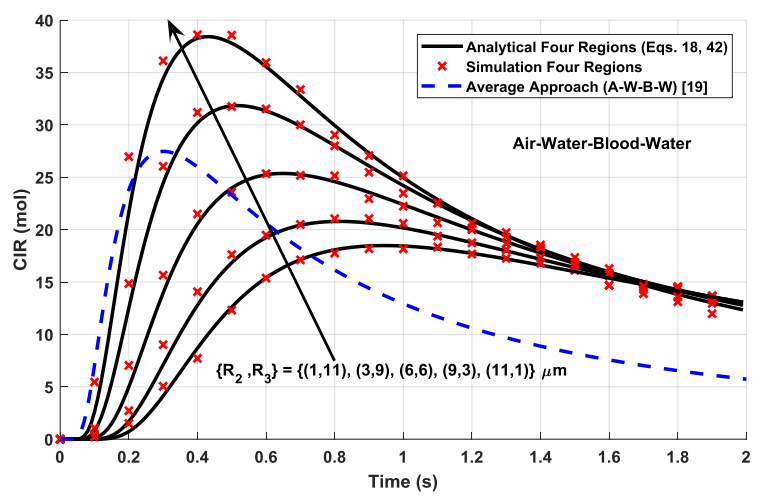

Fig. 8. The CIR as a function of time for four-region medium with different thicknesses of region-2 and region-3. 


\section{CONCLUSIONS}

The paper presents a rigorous analytical formulation to derive a 3-D channel impulse response (CIR) for $\mathrm{MCvD}$ in a composite diffusive medium having multiple regions, which has not been addressed so far in the literature. Closed form, analytical expressions for a 3-D CIR for a general medium with $\mathrm{N}$ multiple regions are derived. Furthermore, we have also demonstrated that the CIR for a multiple region medium reduces to that of a single homogeneous region when the diffusion coefficients of the regions become equal. Three communication metrics viz., the pulse peak amplitude, pulse peak time and pulse width have been introduced for the two region $\mathrm{MCvD}$ channel model. A multiple region medium consisting of air, water, and blood is used to demonstrate the effects of the interface separation, diffusion coefficients, region thickness etc., on the CIR. We have also proposed a generalised particle-based simulator for the 3-D multiple region molecular diffusive channel and validated the simulations for a multiple region channel with the exact results obtained from the analytical formulation.

Our investigations have revealed that, when $\mathrm{TN}$ and $\mathrm{RN}$ are placed in different regions, the diffusion coefficients and the separation distance between either $\mathrm{TN}$ or $\mathrm{RN}$ with respect to the interface $\left(Z_{\text {sep }}, Z_{\text {sep }}^{\prime}\right)$ significantly influence the CIR. However, when both TN and RN are placed in the same region (region1), $\mathrm{CIR}_{1}$ will be mainly affected by $\mathrm{D}_{1}$ and the effect of the interface separation appears to be negligible. In addition, unlike the single region case, the pulse peak amplitude mainly depends on diffusion coefficients when a passive receiver is located in a multiple region medium. We believe that the work presented in this paper will help to enhance the understanding of the multiple region diffusive channel in order to design optimum transmitting and receiving nano-machines as well as equalization and detection techniques.

\section{Appendix A}

Derivation of Eq. (17)

To derive (17), we write (16) as an integral in terms of the Bessel function after substituting $\mu=0$ and $v=0.5$ in [38, eq. (13.47.2)], as follows

$$
\begin{aligned}
\int_{0}^{\infty} J_{0}(\mathrm{bt}) & \frac{K_{1 / 2}\left(a \sqrt{t^{2}+z^{2}}\right)}{\left(t^{2}+z^{2}\right)^{1 / 4}} t d t \\
& =\frac{1}{a^{1 / 2}}\left(\frac{\sqrt{a^{2}+b^{2}}}{z}\right)^{-1 / 2} K_{-1 / 2}\left(z \sqrt{a^{2}+b^{2}}\right)
\end{aligned}
$$

where, $K_{\mathrm{n}}($.$) is the modified Bessel function of second kind of$ $\mathrm{n}^{\text {th }}$ order.

Now, we expressing $K_{(1 / 2)}$ (.) and $K_{(-1 / 2)}$ (.) in (44) using the identities [38, eq. (3.71.13)] and the fact that $K_{(1 / 2)}($.$) and K_{(-1 / 2)}$ (.) are equal as follows

$$
\int_{0}^{\infty} J_{0}(b t) \frac{e^{-a \sqrt{t^{2}+c^{2}}}}{\sqrt{t^{2}+c^{2}}} t d t=\frac{1}{\sqrt{\left(a^{2}+b^{2}\right)}} e^{-c \sqrt{a^{2}+b^{2}}}
$$

Finally, (17) can be obtained by substituting $a=|z-z o|, b=R$, $c=\lambda_{1}$, and $t=\xi$, and then multiplying both sides of (45) by $\exp (-$ $\left.s t_{0}\right) /\left(4 \pi D_{l}\right)$.

\section{Appendix B}

Derivation of Eqs. (22)-(24)

The functions $\mathrm{A}_{2}$ and $\mathrm{B}_{1}$ can be obtained by applying the boundary conditions (8)-(9) for (20)-(21) as follows

$$
\begin{aligned}
& \lim _{z \rightarrow \infty} \int_{0}^{\infty}\left[\frac{e^{-s t_{0}} e^{-g_{1}\left|z-z_{0}\right|}}{4 \pi D_{1} g_{1}}+A_{1} e^{-g_{1} z}+B_{1} e^{g_{1} z}\right] J_{0}(\xi \mathrm{R}) \xi d \xi=0 \\
& \lim _{z \rightarrow-\infty} \int_{0}^{\infty}\left[A_{2} e^{-g_{2} z}+B_{2} e^{g_{2} z}\right] J_{0}(\xi \mathrm{R}) \xi d \xi=0
\end{aligned}
$$

Then, after evaluating the limits in (46)-(47), we get the following equations

$$
\begin{aligned}
& \frac{e^{-s t_{0}}}{4 \pi D_{1}} \frac{1}{g_{1}} e^{-\infty}+A_{1} e^{-\infty}+B_{1} e^{\infty}=0 \\
& A_{2} e^{\infty}+B_{2} e^{-\infty}=0
\end{aligned}
$$

Now, by solving (48)-(49), we conclude that $A_{2}$ and $B_{1}$ should be vanished $\left(A_{2}=B_{1}=0\right)$ and thus we get (22). The functions $A_{1}$ and $\mathrm{B}_{2}$ can be obtained by applying the boundary conditions (6)-(7) for (20)-(21) after substituting $\left(\mathrm{A}_{2}=\mathrm{B}_{1}=0\right)$ as follows

$$
\begin{gathered}
\frac{e^{-s t_{0}}}{4 \pi} e^{g_{1}\left(z_{1}-z_{0}\right)}-A_{1} D_{1} g_{1} e^{-g_{1} z_{1}}=D_{2} B_{2} g_{2} e^{g_{2} z_{1}} \\
\frac{e^{-s t_{0}}}{4 \pi D_{1} g_{1}} e^{g_{1}\left(z_{1}-z_{0}\right)}\left(D_{1} g_{1}-\sigma_{1}\right)-D_{1} A_{1} g_{1} e^{-g_{1} z_{1}} \\
=\sigma_{1}\left(A_{1} e^{-g_{1} z_{1}}-k_{1} B_{2} e^{g_{2} z_{1}}\right)
\end{gathered}
$$

where, the absolute value function in (20) is $\left|z_{1}-z_{0}\right|=-\left(z_{1}-z_{0}\right)$ because we assume that the TN located in the first region (i.e., $\left.z_{1}<z_{0}\right)$ where $z_{1}$ is the location of the interface along z-axis. Finally, (23)-(24) can be obtained by solving (50)-(51) for $A_{1}$ and $\mathrm{B}_{2}$.

\section{Appendix C \\ Derivation of Eq. (31)}

Using the identity [28, eq. (29.3.82)] and Convolution theorem [39, eq. (1.25)] with helping of the time shifting and complex shifting properties of Laplace Transform, the inverse Laplace transform of (30) can be written as follows

$$
\begin{gathered}
f_{3}=\frac{k_{1}}{2 \pi} \int_{0}^{\infty} \int_{0}^{t} \int_{0}^{\infty} \exp \left(-\xi^{2}\left(D_{1}\left(\tau-t_{0}\right)+D_{2}\left(t-\tau-t_{0}\right)\right)\right) \\
\times J_{0}(\xi \mathrm{R}) \xi d \xi \frac{\left(m k_{1} D_{1}+\left(z_{0}+z-2 z_{1}\right)\right)}{2 \sqrt{\pi D_{1}\left(\tau-t_{0}\right)^{3}}} \frac{\mathrm{m} D_{2}}{2 \sqrt{\pi D_{2}\left(t-\tau-t_{0}\right)^{3}}} \\
\times \exp \left(-\frac{\left(m k_{1} D_{1}+\left(z_{0}+z-2 z_{1}\right)\right)^{2}}{4 D_{1}\left(\tau-t_{0}\right)}-\frac{\left(\mathrm{m} D_{2}\right)^{2}}{4 D_{2}\left(t-\tau-t_{0}\right)}\right) d \tau d m
\end{gathered}
$$

Now, using [28, eq. (11.4.29)], (52) can be written as follows 


$$
\begin{aligned}
& f_{3}=\frac{k_{1}}{16 \pi^{2} \sqrt{D_{1} D_{2}}} \int_{0}^{t} \frac{\exp \left(-\frac{R^{2}}{4\left(D_{1}\left(\tau-t_{0}\right)+D_{2}\left(t-\tau-t_{0}\right)\right)}\right)}{\left(\tau-t_{0}\right)^{3 / 2}\left(t-\tau-t_{0}\right)^{3 / 2}\left(D_{1}\left(\tau-t_{0}\right)+D_{2}\left(t-\tau-t_{0}\right)\right)} \\
& \times \int_{0}^{\infty}\left(m^{2} D_{1} D_{2} k_{1}-m D_{2}\left(2 z_{1}-z_{0}-z\right)\right) \\
& \times \exp \left(-m^{2} \frac{\left(k_{1}^{2} D_{1}\left(t-\tau-t_{0}\right)+D_{2}\left(\tau-t_{0}\right)\right)}{4\left(t-\tau-t_{0}\right)\left(\tau-t_{0}\right)}-2 m \frac{-k_{1}\left(2 z_{1}-z_{0}-z\right)}{4\left(\tau-t_{0}\right)}-\right. \\
& \left.\frac{\left(2 z_{1}-z_{0}-z\right)^{2}}{4 D_{1}\left(\tau-t_{0}\right)}\right) d m d \tau
\end{aligned}
$$

The integral w.r.t $\mathrm{m}$ in (53) can be written as follows

$$
\int_{0}^{\infty}\left(b_{1} m^{2}-b_{2} \mathrm{~m}\right) e^{-c_{1} m^{2}-2 m c_{2}-c_{3}} d m
$$

where,

$$
\begin{aligned}
& c_{1}=\frac{\left(k_{1}^{2} D_{1}\left(t-\tau-t_{0}\right)+D_{2}\left(\tau-t_{0}\right)\right)}{4\left(t-\tau-t_{0}\right)\left(\tau-t_{0}\right)}, c_{2}=-\frac{k_{1}\left(2 z_{1}-z_{0}-z\right)}{4\left(\tau-t_{0}\right)}, \\
& c_{3}=\frac{\left(2 z_{1}-z_{0}-z\right)^{2}}{4 D_{1}\left(\tau-t_{0}\right)}, b_{1}=D_{1} D_{2} k_{1}, b_{2}=D_{2}\left(2 z_{1}-z_{0}-z\right)
\end{aligned}
$$

The integral (54) can be evaluated using identities [30, eq. (3.462.5) and eq. (3.462.7)] or directly using Mathematica software as follows

$$
\begin{gathered}
\int_{0}^{\infty}\left(b_{1} m^{2}-b_{2} \mathrm{~m}\right) e^{-c_{1} m^{2}-2 m c_{2}-c_{3}} \quad d m=-\frac{c_{2} b_{1}+c_{1} b_{2}}{2 c_{1}^{2}} e^{-c_{3}} \\
+\frac{\sqrt{\pi} e^{\frac{c_{2}^{2}}{c_{1}} c_{3}}}{4 c_{1}^{3 / 2}}\left(\frac{2 c_{2}^{2} b_{1}}{c_{1}}+b_{1}+2 c_{2} b_{2}\right) \operatorname{erfc}\left(\frac{c_{2}}{\sqrt{c_{1}}}\right)
\end{gathered}
$$

We assume that the TN emits the information molecules initially at time $t_{0}=0$. After substituting (55) in (56) and then (56) in (53) and using the dimensionless parameters (33) and interchange the variable of integration to $v=\tau / t$, we get (31).

\section{REFERENCES}

[1] I. F. Akyildiz, F. Brunetti, and C. Blázquez, "Nanonetworks: A new communication paradigm," Comput. Netw., vol. 52, no. 12, pp. 22602279, Aug. 2008.

[2] T. Nakano, A. W. Eckford, and T. Haraguchi, Molecular communication, Cambridge, UK: Cambridge Univ. Press, 2013.

[3] N. Farsad, H. B. Yilmaz, A. Eckford, C.-B. Chae, and W. Guo, "A comprehensive survey of recent advancements in molecular communication," IEEE Commun. Surveys Tuts., vol. 18, no. 3, pp. 1887 1919, Feb. 2014.

[4] N. Farsad, N.-R. Kim, A. W. Eckford, and C.-B. Chae, "Channel and noise models for nonlinear molecular communication systems," IEEE J. Sel. Areas Commun., vol. 32, no. 12, pp. 2392-2401, Dec. 2014.

[5] P.-C. Yeh, K.-C. Chen, Y.-C. Lee, L.-S. Meng, P.-J. Shih, P.-Y. Ko, W.A. Lin, and C.-H. Lee, "A new frontier of wireless communication theory: diffusion-based molecular communications," IEEE Wirel. Commun., vol. 19 , no. 5, Oct. 2012.

[6] M. Gregori, and I. F. Akyildiz, "A new nanonetwork architecture using flagellated bacteria and catalytic nanomotors," IEEE J. Sel. Areas Commun., vol. 28, no. 4, May 2010.

[7] K. Darchini, and A. S. Alfa, "Molecular communication via microtubules and physical contact in nanonetworks: A survey," Nano Commun. Netw., vol. 4, no. 2, pp. 73-85, Jun. 2013.

[8] H. C. Berg, Random walks in biology, Princeton, NJ: Princeton Univ. Press, 1993.

[9] V. Jamali, A. Ahmadzadeh, C. Jardin, H. Sticht, and R. Schober, "Channel Estimation for Diffusive Molecular Communications," IEEE Trans. Commun., vol. 64, no. 10, pp. 4238-4252, Oct. 2016.
[10] A. Noel, K. C. Cheung, and R. Schober, "Optimal receiver design for diffusive molecular communication with flow and additive noise," IEEE Trans. Nanobiosci., vol. 13, no. 3, pp. 350-362, Jul. 2014.

[11] I. Llatser, A. Cabellos-Aparicio, M. Pierobon, and E. Alarcón, "Detection techniques for diffusion-based molecular communication," IEEE J. Sel. Areas Commun., vol. 31, no. 12, pp. 726-734, Dec. 2013.

[12] A. Gohari, M. Mirmohseni, and M. Nasiri-Kenari, "Information Theory of Molecular Communication: Directions and Challenges," IEEE Trans. Mol. Biol. Multi-Scale Commun. , vol. 2, no. 2, pp. 120-142, Dec. 2016.

[13] H. Lodish, D. Baltimore, A. Berk, S. L. Zipursky, P. Matsudaira, and J. Darnell, Molecular cell biology, 3rd ed., New York: Scientific American Books, 1995.

[14] A. Guyton, and J. Hall, Textbook of Medical Physiology, 11 ed., Philadelphia: WB Saunders Company, 2006.

[15] B. K. Lawther, S. Kumar, and H. Krovvidi, "Blood-brain barrier," Cont. Edu. Anaes., Criti. Care \& Pain, vol. 11, no. 4, pp. 128-132, Jun. 2011.

[16] S. McGinty, "A decade of modelling drug release from arterial stents," Math. Biosci. ， vol. 257, pp. 80-90, Nov. 2014.

[17] Y. Chahibi, and I. F. Akyildiz, "Molecular communication noise and capacity analysis for particulate drug delivery systems," IEEE Trans. Commun., vol. 62, no. 11, pp. 3891-3903, Nov. 2014.

[18] R. Edmondson, J. J. Broglie, A. F. Adcock, and L. Yang, "Threedimensional cell culture systems and their applications in drug discovery and cell-based biosensors," Assay Drug Dev. Techn., vol. 12, no. 4, pp. 207-218, May 2014.

[19] S. M. Mustam, S. Yusof, S. Kamilah, and S. Nejatian, "Multilayer diffusion-based molecular communication," Trans. Emerg. Telecommun. Tech., vol. 28, no. 1, Feb. 2015.

[20] S. M. Mustam, S. K. Syed-Yusof, and S. Zubair, "Capacity and Delay Spread in Multilayer Diffusion-Based Molecular Communication (DBMC) Channel," IEEE Trans. Nanobiosci., vol. 15, no. 7, pp. 599-612, Oct. 2016.

[21] J. Crank, The mathematics of diffusion, 2 ed., Oxford, UK: Oxford Univ. Press, 1980.

[22] I. Llatser, D. Demiray, A. Cabellos-Aparicio, D. T. Altilar, and E. Alarcón, "N3Sim: Simulation framework for diffusion-based molecular communication nanonetworks," Simul. Model. Pract. Th., vol. 42, pp. 210-222, Mar. 2014.

[23] M. Pierobon, and I. F. Akyildiz, "A statistical-physical model of interference in diffusion-based molecular nanonetworks," IEEE Trans. Commun., vol. 62, no. 6, pp. 2085-2095, Jun. 2014.

[24] M. Burgener, and R. Reedy, "Temperature distributions produced in a two-layer structure by a scanning cw laser or electron beam," J. Appl. Phys., vol. 53, no. 6, pp. 4357-4363, Feb. 1982.

[25] D. Kilinc, and O. B. Akan, "Receiver design for molecular communication," IEEE J. Sel. Areas Commun., vol. 31, no. 12, pp. 705714, Dec. 2013.

[26] M. U. Mahfuz, D. Makrakis, and H. T. Mouftah, "A comprehensive study of sampling-based optimum signal detection in concentration-encoded molecular communication," IEEE Trans. Nanobiosci., vol. 13, no. 3, pp. 208-222, Aug. 2014.

[27] T. Koizumi, M. Kakemi, K. Katayama, and H. Ohtani, "A note on diffusion in finite composite media," Chem. Pharm. Bull. , vol. 31, no. 3, pp. 1092-1096, 1983.

[28] M. Abramowitz, and I. A. Stegun, Handbook of mathematical functions: with formulas, graphs, and mathematical tables: Courier Corporation, 1964.

[29] A. Noel, K. C. Cheung, and R. Schober, "Using dimensional analysis to assess scalability and accuracy in molecular communication," Proc. IEEE ICC MONACOM, pp. 818-823, Jun. 2013.

[30] A. Jeffrey, and D. Zwillinger, Table of integrals, series, and products: Academic press, 2007.

[31] Viscopedia.com, "A free encyclopedia for viscosity."

[32] N.-R. Kim, A. W. Eckford, and C.-B. Chae, "Symbol interval optimization for molecular communication with drift," IEEE Trans. Nanobiosci., vol. 13, no. 3, pp. 223-229, Sept. 2014.

[33] R. A. Freitas Jr, Nanomedicine, volume I: Basic Capabilities., Georgetown, TX: Landes Bioscience, 1999.

[34] Y. Lu, M. D. Higgins, A. Noel, M. S. Leeson, and Y. Chen, "The effect of two receivers on broadcast molecular communication systems," IEEE Trans. Nanobiosci., vol. 15, no. 8, pp. 891-900, Oct. 2016.

[35] B.-H. Koo, C. Lee, H. B. Yilmaz, N. Farsad, A. Eckford, and C.-B. Chae, "Molecular MIMO: From theory to prototype," IEEE J. Sel. Areas Commun., vol. 34, no. 3, pp. 600-614, Mar. 2016. 
[36] H. B. Yilmaz, A. C. Heren, T. Tugcu, and C.-B. Chae, "Threedimensional channel characteristics for molecular communications with an absorbing receiver," IEEE Commun. Lett., vol. 18, no. 6, pp. 929-932, Jun. 2014.

[37] M. Ş. Kuran, H. B. Yilmaz, T. Tugcu, and B. Özerman, "Energy model for communication via diffusion in nanonetworks," Nano Commun. Net., vol. 1, no. 2, pp. 86-95, Jun. 2010.

[38] G. N. Watson, A treatise on the theory of Bessel functions, Cambridge, UK: Cambridge University Press, 1995.

[39] A. M. Cohen, Numerical methods for Laplace transform inversion, NY: Springer Science \& Business Media, 2007. [40] R. G. Rice and D. D. Do, Applied Mathematics and Modeling for Chemical Engineers, NY: Wiley, pp. 383-385,1995.

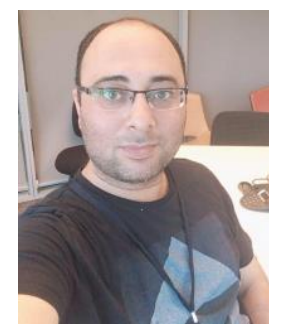

Muneer M. Al-Zu'bi received the M.Sc. and B.Sc degrees in electrical Engineering with First Class Honours [H1] from Jordan University of Science and Technology (JUST) and Al- Balqa' Applied University (BAU), Jordan, in 2014 and 2010, respectively. He is currently pursuing the Ph.D. degree with the School of Biomedical Engineering, University of Technology Sydney (UTS). He served as a research assistant on many research projects with the Jordan University of Science and Technology (JUST), 2014-2015. He also served as a communication engineer with KADDB for production of military equipments, 2016, Jordan. He was the recipient of many scholarships during his B.Sc., M.Sc., and Ph.D. studies. He designed many innovation projects using hardware and software in communication, computer, and biomedical engineering. His main research interests are in the areas of molecular communications, biomedical wireless communications, bionano machines, nano communication, and EM wave propagation.

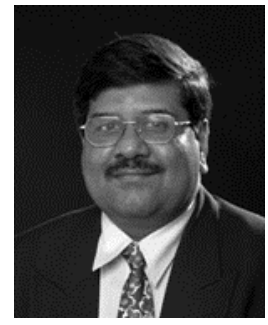

Ananda Mohan Sanagavarapu (aka 'Ananda S Mohan') (SM'05) received the Ph.D. degree from the Indian Institute of Technology, Kharagpur, India. $\mathrm{He}$ is currently an Associate Professor in the recently established School of Biomedical Engineering, Faculty of Engineering and Information Technology, University of Technology Sydney (UTS), NSW, Australia, where he leads research on Microwave Imaging, Molecular Communications, $\mathrm{RF}$ and Microwave Thermal Therapies, Implantable and Ingestible Biomedical Devices, Array Signal Processing, Wireless Biomedical Communications and Applied Electromagnetics. He is a founding core member of the Interdisciplinary Research Centre on Health Technologies at UTS. He was formerly the Co-Director of the UTS branch of Sydney Microwave Design Resource Centre and also an Associate Director of the UTS node of the multi university Cooperative Research Centre on Satellite Systems. Before joining UTS, he held a postdoctoral fellowship at the Air Navigation Research Group, School of Electrical Engineering at the University of Sydney, Australia. He received a number of competitive research grants from Australian Research Council, National Health and Medical Research Council, and industry. $\mathrm{He}$ has also mentored large number of $\mathrm{PhD}$ and masters (research) students. Prof. Mohan was a co-recipient of the Priestly Memorial Award from the Institute of Radio and Electronic Engineers, Australia. He was a member of the IEEE in New South Wales Section Committee and was the past chair of IEEE NSW AP/MTT joint chapter and also a past committee member of NSW IEEE Communications and Signal Processing Society chapter. He was member of Technical Program Committee of IEEE Globecom-98, a publicity co-chair of the 2011 Asia Pacific Microwave Conference and a Technical Program Committee Co-Chair for iWAT2014 and ISAP2015. 